\title{
Determination of Harmonic Parameters with Temporal Variations: An Enhanced Harmonic Analysis Algorithm and Application to Internal Tidal Currents in the South China Sea
}

\author{
GUANGZHEN JIN \\ School of Marine Sciences, Sun Yat-Sen University, and Key Laboratory of Marine Resources and Coastal Engineering in Guangdong \\ Province, Guangzhou, and Physical Oceanography Laboratory/Qingdao Collaborative Innovation Center of Marine Science and \\ Technology, Ocean University of China, and Qingdao National Laboratory for Marine Science and Technology, Qingdao, China
}

\author{
Haidong Pan, Qilin Zhang, Xianqing Lv, And Wei ZhaO
}

Physical Oceanography Laboratory/Qingdao Collaborative Innovation Center of Marine Science and Technology, Ocean University of China, and Qingdao National Laboratory for Marine Science and Technology, Qingdao, China

YUAN GAO

Department of Mathematics, Hong Kong University of Science and Technology, Kowloon, Hong Kong, China

(Manuscript received 12 December 2016, in final form 28 February 2018)

\begin{abstract}
As an effective tool to distinguish different tidal components, classical tidal current harmonic analysis has been widely used to obtain harmonic parameters of internal tidal currents. However, harmonic parameters cannot exactly reveal the motion of internal tides, as the irregular temporal variations for internal tides are significant in many regions of the world's oceans. An enhanced harmonic analysis (EHA) algorithm based on the independent point scheme and cubic spline interpolation is presented in this paper to obtain harmonic parameters with temporal variations for different tidal constituents of internal tides. Moreover, this algorithm is applied to analyze 14 months of current data obtained from a mooring located on the continental shelf in the northeastern region of the South China Sea. The obvious irregular temporal variations for the four principal constituents $-\mathrm{M}_{2}, \mathrm{~K}_{1}, \mathrm{~S}_{2}$, and $\mathrm{O}_{1}-$ of internal tides in this region are indicated. It is hoped that this algorithm might present a brand-new view for researchers to investigate the irregular temporal motions of internal tides.
\end{abstract}

\section{Introduction}

Over centuries great progress has been made to explain and predict oceanic tides. Our understanding of tides deepened with several breakthroughs made by some scientific pioneers (e.g., Newton's theory of gravitation and equilibrium tide, and Laplace's expression about the tidal potential). Internal tides - namely, internal waves with tidal frequency-were first observed by Helland-Hansen and Nansen (1909) in the Norwegian Sea by means of hydrographic observations. Since then they have been observed

Supplemental information related to this paper is available at the Journals Online website: https://doi.org/10.1175/JTECH-D-16-0239.s1.

Corresponding author: Xianqing Lv, xqinglv@ouc.edu.cn; Wei Zhao,weizhao@ouc.edu.cn in many places in the world's oceans (Baines 1973). There is an increasing need to understand the dynamic behavior of oceanic internal tides and internal tidal currents.

The harmonic method for tidal analysis is an effective tool to distinguish different tidal components and is widely used in the study of oceanic tides or tidal current and internal tides or internal tidal currents. With a least squares fitting method, the harmonic analysis (HA) can be used to determine the relative amplitude and phase lag of each tidal component. Because traditional ordinary least squares minimization is highly sensitive to the nontidal components in the observed signal that will introduce nontidal noise into the tidal signal by recording and transcription errors and climatic events, essentially the least squares minimization overfits the nontidal components in an attempt to minimize the total residual error (Leffler and Jay 2009) and obtains constants (phase 
and amplitude) for each tidal frequency. This may be practicable in the study of oceanic tides and tidal currents because surface tides in the deep ocean are nearly periodic and predictable at most locations (Matte et al. 2013). However, there are many situations in which the observed tides or tidal currents are modulated by some nontidal processes, some of which occur in the tidal frequency band. Also, morphological modifications leading to changes in bed friction, surface slope, and/or vegetation may alter tidal properties (Amin 1983, 1985; Godin 1985; DiLorenzo et al. 1993; Horsburgh and Wilson 2007; Jay 2009; Jay et al. 2011). Some scientists have noticed the issue and carried out research on generalizing classical harmonic analysis to allow for the study of nonstationary tides (Amin 1983, 1985, 1993; Jay and Flinchem 1995, 1997, 1999; Jay 1991, 2009; Jay and Kukulka 2003; Buschman et al. 2009; Kukulka and Jay 2003a,b; Parker 2007; Matte et al. 2013; Zaron and Jay 2014; Pan et al. 2018). Most of these inspiring works, however, were applied to the problem of river tides, because they are "conceptually the simplest non-stationary tidal process" (Jay and Flinchem 1997, p. 5705). But when it comes to internal tides and internal tidal currents, things are quite more complicated and many researchers have found evidence for the irregular temporal variations of internal tides (van Haren et al. 1999; Gerkema et al. 2004; van Haren 2004; Jan et al. 2008; Shaw et al. 2009; Buijsman et al. 2010; Klymak et al. 2011; Lee et al. 2012; Wu et al. 2013; Alford et al. 2015; Kelly et al. 2015; van Haren et al. 2015). As a consequence of the seasonal variations in stratification, the generation and propagation of internal tides show irregular variations that are not phase locked (incoherent) with astronomical forcing (Lee et al. 2012). To the best of our knowledge, most studies on internal tidal analysis employed classical harmonic analysis (Guo et al. 2006; Plueddemann and Farrar 2006; Teague et al. 2007; Duda and Rainville 2008; Zu et al. 2008; Liu et al. 2010; Ramp et al. 2010; Zhao et al. 2010; Xu et al. 2011; J. Zhang et al. 2011; Guo et al. 2012; Lee et al. 2012; Xu et al. 2013; Gao et al. 2015; Gao et al. 2016; Xu et al. 2016), which may provide only approximate values of tidal constituent properties for the nonstationary signal.

Limited in situ observations and results from numerical models have provided some hints on this issue, but more research is needed to investigate the phenomenon of the irregular temporal variations of internal tides and internal tidal currents. In this work changes are made for the classical harmonic analysis (CHA) algorithm to make the method suitable for the analysis of nonstationary signals. We rethink the concept of harmonic constants because constituent amplitudes and phases will be defined as timedependent values. Then the traditional Rayleigh criterion is employed as a reference for users to decide which constituents to be included for the harmonic analysis.
Finally, we present an enhanced harmonic analysis (EHA) algorithm using an independent point scheme to reduce the number of undetermined harmonic parameters to obtain a more accurate result. The mean difference between EHA and the segmented harmonic analysis with cubic spline interpolation lies in the fact that the former is using the data from the entire record to determine the harmonic parameters at any particular point with the spline coupling included.

This paper is organized as follows. Section 2 presents the main methodology of the EHA algorithm. Then the capability of EHA is investigated in section 3 with a numerical example. In section 4 EHA is applied to 14-month mooring current data in the South China Sea (SCS) and harmonic parameters with significant temporal variations are obtained. Finally, the summary of this paper is given in section 5 .

\section{Methodology}

\section{a. Statistical model for EHA}

Harmonic tidal current analysis is usually conducted to obtain the constants of amplitudes and phase lags for each prescribed tidal frequency. Originating in the late nineteenth century, the traditional harmonic tidal model involving tidal and nontidal energies can be expressed as

$$
\begin{aligned}
h\left(t_{i}\right) & =S_{0}+\sum_{j=1}^{J} A^{j} \times \cos \left(\sigma^{j} t_{i}-\phi^{j}\right)+R\left(t_{i}\right), \text { for } \\
i & =0,1, \ldots, N
\end{aligned}
$$

where $h\left(t_{i}\right)$ is the measurement at time $t_{i} ; S_{0}$ is a constant datum; $A_{j}, \phi^{j}$, and $\sigma^{j}(j=1, J)$ are the amplitude, phase lag, and tidal frequency of constituent $j$, respectively; $R\left(t_{i}\right)$ is the nontidal residual (Foreman et al. 2009); $J$ is the number of tidal constituents; and $N$ is the number of observation time samples.

The HA approach was first proposed by Darwin in the nineteenth century (Darwin 1891). In mathematical terms this HA approach is implemented by Foreman (1977) employing FORTRAN 77 and by Pawlowicz et al. (2002) employing MATLAB. Codiga (2011) then improves the tidal program T_TIDE (Pawlowicz et al. 2002) in many respects, including the advances of Leffler and Jay (2009) and the second Foreman code (Foreman et al. 2009), in a MATLAB code. Though it has been applied to research in different fields for many years (Foreman et al. 2009), the foremost limitation of this approach lies in the assumption of stationarity. This assumption works well on the traditional harmonic analysis for water elevations within short periods (e.g., one year or so) and tidal-tidal current data within a short period (e.g., one year or so). However, the conventional HA does not work particularly well for current data 
because of the nonstationary (Godin 1983). Moreover, when it comes to the cases in which the nonlinear interactions play an important role, such an assumption is often invalid. In the first case of shallow water, as a result of nonlinear interactions between the tide and storm surges or variable river discharges, the tidal amplitudes and phases are changed during periods when these phenomena occur (Foreman et al. 2009). A series of works by David Jay and colleagues (Jay 1991, 2009; Jay and Flinchem 1995, 1997, 1999; Jay et al. 2011; Kukulka and Jay 2003a,b; Matte et al. 2013) draw a clear map on the harmonic analysis of nonstationary river tides. In the second case of secular recorded tides, as has been discovered by means of wavelet analysis (Jay and Flinchem 1997, 1999; Jay 2009), the surface expressions of internal tides constitute a significant component of the total recorded tide (Colosi and Munk 2006). As early as the 1960s, Munk and Bullard (1963, p. 344) suggested that "the mean sea level may vary by $10 \mathrm{~cm}$ in a decade, and the tidal constants are altered by this variation." Actually, fluctuations in sea level in the frequency range of cycles per decade to cycles per day are associated, apart from the tides (Groves and Zetler 1964; Munk and Bullard 1963). Based on the venerable tidal records in the Honolulu tidal gauge, Colosi and Munk (2006) presented the variability of surface tide modulated by the $\mathrm{M}_{2}$ internal tide, which highly inspired this paper. In fact, there may be some possibilities that internal tides are modulated by the slowly varying thermal structure (Colosi and Munk 2006), the change of stratification or seasonally varying ice cover (Foreman et al. 2009), and the nonlinear interactions between them and background internal waves. Until recently, various researchers (Gerkema et al. 2004; Jan et al. 2008; Shaw et al. 2009; Buijsman et al. 2010; Klymak et al. 2011) have indicated the existence of irregular temporal variations for internal tides inside the ocean by viewing the in situ observations. In an effort to obtain time-variable tidal harmonic parameters, an enhanced harmonic analysis algorithm has been developed. We will first introduce a statistical model based on the classical tidal harmonic analysis to nonstationary internal tidal records in order to distinguish between the time-variable tidal harmonic parameters and the nontidal components:

$$
\begin{aligned}
h\left(t_{i}\right) & =S_{0}\left(t_{i}\right)+\sum_{j=1}^{J} A^{j}\left(t_{i}\right) \cos \left[\sigma^{j} t_{i}-\phi^{j}\left(t_{i}\right)\right]+R\left(t_{i}\right), \text { for } \\
i & =0,1, \ldots, N .
\end{aligned}
$$

The main difference between this model and the traditional one is the assumption of nonstationary amplitude $A^{j}(t)$, phase lag $\phi^{j}(t)$ of constituent $j$, and the zero-frequency component $S_{0}(t)$. Besides, the "noise" $R\left(t_{i}\right)$ for the time series may contain the nontidal residual and the

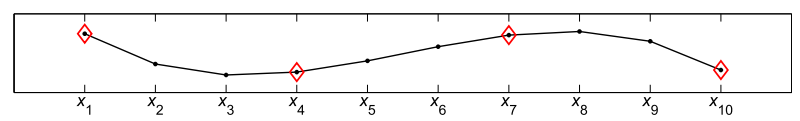

FIG. 1. Sketch illustrates IPS. The $x$ axis has labels of $x_{1}$ on the left to $x_{10}$ on the right.

variability of tidal parameters at time scales that cannot be resolved. To obtain the harmonic parameters of internal tides with temporal variations [i.e., $A^{j}(t), \phi^{j}(t)$, and $S_{0}(t)$ in Eq. (2)], we present the EHA algorithm that introduces an independent point scheme (see section 2b) and the cubic spline interpolation (see section 2c) to determine continuous and smooth temporal variation of amplitudes and phases for a specific tidal component.

\section{b. Independent point scheme and cubic spline interpolation}

Harmonic analysis is a kind of inverse problem to determine the unknown harmonic parameters with observed tidal (current) data. In practice, the number of constituents chosen needs to be commensurate with the length of record and the noise level. Additionally, the harmonic results obtained by segmented harmonic analysis are independent of each other. The independent point scheme is a kind of methodology to connect unresolved parameters in the methodology of harmonic analysis. As a result, it will lead to continuous harmonic results directly rather than manual interpolations. The philosophy of the independent point scheme (IPS) has been put forward in some studies to deal with the ill-posedness of the inverse problem, for 1D (Zhang and Lu 2010; Gao et al. 2013) and 2D parameters (Lu and Zhang 2006; J. Zhang et al. 2011; Jin et al. 2015). Readers who are interested in this issue are referred to Pan et al. (2017) and Guo et al. (2017) for more details. The basic idea of IPS is quite simple; see Fig. 1 for instance. Assume the harmonic parameters (e.g., amplitude $H$ ) are varying in time and $I=\left\{x_{1}, x_{2}, \ldots, x_{10}\right\}$ are the time indices. First, the indices of independent points (IPs) $\widetilde{I}=\left\{x_{1}, x_{4}, x_{7}, x_{10}\right\}$ are a subset of $I$, which are the representatives of the parameter space. For convenience, these IPs are selected uniformly in this paper. Second, the harmonic parameters at IPs (red rhombuses in Fig. 1) can be calculated through a specific algorithm. Finally, harmonic parameters on the other points (i.e., $x_{2}, x_{3}, x_{5}, x_{6}, x_{8}$, and $x_{9}$ ) can be interpolated between the IPs. Thus, IPS is a helpful tool to reduce the amount of computations and to connect adjacent parameters in the space domain. If a different number of IPs is chosen, then they are regarded as different IPSs.

Here we use cubic splines to interpolate between the IPs because there are two main advantages of this method. One is that the interpolation results can be a 
pleasingly smooth curve (https://mse.redwoods.edu/darnold/ math45/laproj/Fall98/SkyMeg/Proj.PDF). Another advantage of cubic spline interpolation is the undetermined function, and its first two derivatives are continuous. In this case, we will have a tridiagonal linear system that is strictly row diagonally dominant and that can easily find the solution without any difficulty by Gauss elimination (de Boor 1978). In practice, cubic spline has been widely used in different research fields (Schoenberg 1964; Reinsch 1967; de Boor 1978; Wahba 1985; Desquilbet and Mariotti 2010; Lawn et al. 2011; Malik et al. 2011; Hu et al. 2012; The 1000 Genomes Project Consortium 2015). Because of the Rayleigh criterion, it is almost impossible to obtain harmonic parameters on all sample points. Thus, temporal variations for the internal tidal harmonic parameters are usually difficult to analyze. As a result, any function that would effectively correlate all the parameters would be difficult to obtain and be highly unwieldy. So, a series of unique cubic polynomials are fitted between each of the IPs, with the stipulation that the curve obtained be continuous and appear smooth (https://mse.redwoods.edu/darnold/math45/laproj/ Fall98/SkyMeg/Proj.PDF).

\section{c. Enhanced harmonic analysis}

The CHA algorithm, employing the least squares fit, treats the amplitude and phase of a tidal constituent and the mean tidal current as constants for the entire sample time. In contrast, EHA treats them as functions of time. Let us first introduce some notations. Assume $N \geq n>0$ are positive integers. Let $\{i ; i=0,1, \ldots, N\}$ be a sequence and $\left\{i_{k} ; k=0,1, \ldots, n\right\}$ be the subsequence of $\{i\}$. From now on we denote $\left\{t_{i} ; i=0,1, \ldots, N\right\}$ as the time index of the whole time domain. Then $\left\{t_{t_{k}} ; k=0,1, \ldots, n\right\}$, the subsequence of $\left\{t_{i}\right\}$, is the time index of IPs. Without confusion, we denote $\tilde{t}_{k} \triangleq t_{i_{k}}$ in the following paragraphs. The main idea of EHA can be expressed in the following steps.

Step 1. Several points in the parameter space with the time index (tilde) of harmonic parameters are selected as IPs. The harmonic parameters at IPs for a specific tidal constituent are $\left\{A\left(\tilde{t}_{k}\right), \phi\left(\tilde{t}_{k}\right), S_{0}\left(\tilde{t}_{k}\right)\right.$; $k=0,1, \ldots, n\}$. According to IPS, the time-varying harmonic parameters $A\left(t_{i}\right)$ and $\phi\left(t_{i}\right)$ for a specific tidal constituent, where $i=1,2, \ldots, N$ can be interpolated as a function of $A\left(\tilde{t}_{k}\right)$, and $\phi\left(\tilde{t}_{k}\right)$, the overall mean tidal flow $S_{0}\left(t_{i}\right)$ can be interpolated as a function of $S_{0}\left(\tilde{t}_{k}\right)$, which are

$$
\begin{aligned}
\left\{A\left(t_{i}\right) ; i=0,1, \ldots, N\right\} & =\mathbf{F}\left[t_{i} ; A\left(\tilde{t}_{1}\right), A\left(\tilde{t}_{2}\right), \ldots, A\left(\tilde{t}_{n}\right)\right], \\
\left\{\phi\left(t_{i}\right) ; i=0,1, \ldots, n\right\} & =\mathbf{F}\left[t_{i} ; \phi\left(\tilde{t}_{1}\right), \phi\left(\tilde{t}_{2}\right), \ldots, \phi\left(\tilde{t}_{n}\right)\right], \quad \text { and } \\
\left\{S_{0}\left(t_{i}\right) ; i=0,1, \ldots, n\right\} & =\mathbf{F}\left[t_{i} ; S_{0}\left(\tilde{t}_{1}\right), S_{0}\left(\tilde{t}_{2}\right), \ldots, S_{0}\left(\tilde{t}_{n}\right)\right],
\end{aligned}
$$

where $\mathbf{F}\left(t_{i}\right)$ denotes the interpolation method and is a matrix with respect to the time index. To ensure the smoothness of the time variation for the amplitude and phase, the cubic spline interpolation method is employed as the $\mathbf{F}\left(t_{i}\right)$ in this paper.

Step 2. The EHA algorithm finds time-dependent $\left\{A\left(t_{i}\right)\right.$, $\left.\phi\left(t_{i}\right), S_{0}\left(t_{i}\right) ; i=0,1, \ldots, N\right\}$ by interpolation from their values at the IPSs. Instead of dealing with harmonic parameters on all the time indices, we present the EHA algorithm in which $A\left(t_{i}\right), \phi\left(t_{i}\right)$, and $S_{0}\left(t_{i}\right)$ are interpolated with $A\left(\tilde{t}_{k}\right), \phi\left(\tilde{t}_{k}\right)$, and $S_{0}\left(\tilde{t}_{k}\right)$, respectively, based on step 1 . As a result, the number of unknown variables will be $(2 J+1) \times(n+1)$ in EHA, where $J$ is the total number of tidal constituents. Similar to the process of CHA, the least squares fit method will be employed to derive the harmonic parameters on IPs, which are $A\left(\tilde{t}_{k}\right), \phi\left(\tilde{t}_{k}\right)$, and $S_{0}\left(\tilde{t}_{k}\right)$. Then the time-varying harmonic parameters- $A\left(t_{i}\right), \phi\left(t_{i}\right)$, and $S_{0}\left(t_{i}\right)$-can be interpolated by means of step 1 . The detailed processes of the two steps will be presented in the following two subsections.

\section{1) DERIVATION OF FORMULAS FOR THE TIME- VARYING TIDAL (CURRENT) CONSTANTS}

In this part, $\mathbf{F}\left(t_{i}\right)$ from $\left[A\left(\tilde{t}_{k}\right), \phi\left(\tilde{t}_{k}\right), S_{0}\left(\tilde{t}_{k}\right)\right]$ to $\left[A\left(t_{i}\right)\right.$, $\left.\phi\left(t_{i}\right), S_{0}\left(t_{i}\right)\right]$ are derived with the cubic spline interpolation method. In the following derivations, as an example of the interpolation process, one of the harmonic parameters $\left[A\left(t_{i}\right), \phi\left(t_{i}\right), S_{0}\left(t_{i}\right)\right]$ will be denoted as $H_{i}=\left\{f\left(t_{i}\right) ; i=\right.$ $0,1, \ldots, N\}$ and one of $\left[A\left(\tilde{t}_{k}\right), \phi\left(\tilde{t}_{k}\right), S_{0}\left(\tilde{t}_{k}\right)\right]$ will be denoted as $\tilde{H}_{k}=\left\{f\left(\tilde{t}_{k}\right) ; k=0,1, \ldots, n\right\}$ The detailed derivations can be found in any reference book or paper on the cubic spline method (de Boor 1978; https://mse. redwoods.edu/darnold/math45/laproj/Fall98/SkyMeg/ Proj.PDF); so, interested readers may refer to the appendix. Finally, for a specific $t \in\left[\tilde{t}_{k}, \tilde{t}_{k+1}\right]$, the cubic spline transformation from $\tilde{H}$ to $\mathbf{H}$ can be expressed as

$$
\mathbf{H} \triangleq \mathbf{F} \cdot \tilde{H}
$$

where $\mathbf{F}$ is the defined transition matrix from $\tilde{H}$ to $\mathbf{H}$ with $n$ rows and $n+1$ columns. 
2) DETERMINATION OF THE TIME-VARYING TIDAL (CURRENT) PARAMETERS WITH THE HARMONIC ANALYSIS METHOD

In this part the time-varying tidal (current) parameters $f(t)$ will be determined using the least squares fitting method. For traditional harmonic analysis, assume that a selection procedure has chosen $J$ constituents for inclusion in the analysis. The next stage is to calculate the amplitude and phase for the sinusoids representing each constituent cluster. The basic methodology for EHA is similar to CHA. For a one-dimensional time series, the analysis objective is to solve the unknown variables $S_{0}\left(t_{i}\right), A^{j}\left(t_{i}\right)$, and $\phi^{j}\left(t_{i}\right)$ in the following system of equations:

$$
\begin{aligned}
h\left(t_{i}\right) & =S_{0}\left(t_{i}\right)+\sum_{j=1}^{J} A^{j}\left(t_{i}\right) \cos \left[\sigma^{j} t_{i}-\phi^{j}\left(t_{i}\right)\right]+r \text { for } \\
i & =0,1, \ldots, N .
\end{aligned}
$$

Here $h\left(t_{i}\right)$ is the observation at time $t_{i}, S_{0}\left(t_{i}\right)$ is the mean flow at time $t_{i}$, and $\sigma^{j}$ is the frequency of constituent $j$; $A^{j}\left(t_{i}\right)$ and $\phi^{j}\left(t_{i}\right)$ are the amplitude and phase of constituent $j$ at time $t_{i}$, respectively; $r$ is the observation noise; and $N$ is the total number of observation time samples. For convenience, we define

$$
x^{j}(t) \triangleq A^{j}(t) \times \cos \left[\phi^{j}(t)\right], \quad y^{j}(t) \triangleq A^{j}(t) \times \sin \left[\phi^{j}(t)\right],
$$

and

$$
\mathbf{Q} \triangleq(\overbrace{\mathbf{F}_{0}, \mathbf{F}_{0}, \ldots, \mathbf{F}_{0}}^{(N+1) / n} ; \overbrace{\mathbf{F}_{1}, \mathbf{F}_{1}, \ldots, \mathbf{F}_{1}}^{(N+\ldots . . ;) / n} \overbrace{\mathbf{F}_{n-1}, \mathbf{F}_{n-1}, \ldots, \mathbf{F}_{n-1}}^{(N+1) / n})^{\mathrm{T}} \triangleq\left[c_{0}\left(t_{i}\right), c_{1}\left(t_{i}\right), \ldots, c_{n}\left(t_{i}\right)\right]
$$

for $i=0,1, \ldots, N$. Term $\mathbf{Q}$ is an extended matrix of $\mathbf{F}$ with $N+1$ rows and $n+1$ columns, and $c_{k}\left(t_{i}\right)$ is the $(k+1)$ th column of $\mathbf{Q}$. Now we can apply the results in section $2 \mathrm{c}(1)$. Using Eq. (5), we can get $\left\{x^{j}\left(t_{i}\right) ; i=0,1, \ldots, N\right\}=\mathbf{Q}\left\{x^{j}\left(\tilde{t}_{k}\right) ; k=0,1, \ldots, n\right\}$. Putting them into Eq. (4), we can obtain

$$
h\left(t_{i}\right)=\mathbf{Q}_{i} \times \mathbf{S}_{0}\left(\tilde{t}_{k}\right)^{T}+\sum_{j=1}^{J}\left\{\mathbf{Q}_{i} \cdot\left[\mathbf{x}^{j}\left(\tilde{t}_{k}\right)\right]^{\mathrm{T}} \cos \left(\sigma^{j} t_{i}\right)+\mathbf{Q}_{i} \cdot\left[\mathbf{y}^{j}\left(\tilde{t}_{k}\right)\right]^{\mathrm{T}} \sin \left(\sigma^{j} t_{i}\right)\right\}+r, \text { for } \quad i=0,1, \ldots, N,
$$

where $\mathbf{Q}_{i}$ denotes the $(i+1)$ th row in matrix $\mathbf{Q}$.
The solution for Eqs. (6) can be obtained as follows. Naturally, for observation time $t_{i}(i=0,1,2, \ldots, N)$, Eqs. (6) can be written as

$$
\left\{\begin{array}{l}
\sum_{k=0}^{n}\left[c_{k}\left(t_{0}\right) S_{0}\left(\tilde{t}_{k}\right)\right]+\sum_{j=1}^{J}\left[c_{0}\left(t_{0}\right) x^{j}\left(\tilde{t}_{0}\right)+c_{1}\left(t_{0}\right) x^{j}\left(\tilde{t}_{1}\right)+\cdots+c_{n}\left(t_{0}\right) x^{j}\left(\tilde{t}_{n}\right)\right] \cos \left(\sigma^{j} t_{0}\right)+ \\
+\sum_{j=1}^{J}\left[c_{0}\left(t_{0}\right) y^{j}\left(\tilde{t}_{0}\right)+c_{1}\left(t_{0}\right) y^{j}\left(\tilde{t}_{1}\right)+\cdots+c_{n}\left(t_{0}\right) y^{j}\left(\tilde{t}_{n}\right)\right] \sin \left(\sigma^{j} t_{0}\right)+r=h\left(t_{0}\right) \\
\vdots \\
\sum_{k=0}^{n}\left[c_{k}\left(t_{N}\right) S_{0}\left(\tilde{t}_{k}\right)\right]+\sum_{j=1}^{J}\left[c_{0}\left(t_{N}\right) x^{j}\left(\tilde{t}_{0}\right)+c_{1}\left(t_{N}\right) x^{j}\left(\tilde{t}_{1}\right)+\cdots+c_{n}\left(t_{N}\right) x^{j}\left(\tilde{t}_{n}\right)\right] \cos \left(\sigma^{j} t_{N}\right)+ \\
+\sum_{j=1}^{J}\left[c_{0}\left(t_{N}\right) y^{j}\left(\tilde{t}_{0}\right)+c_{1}\left(t_{N}\right) y^{j}\left(\tilde{t}_{1}\right)+\cdots+c_{n}\left(t_{N}\right) y^{j}\left(\tilde{t}_{n}\right)\right] \sin \left(\sigma^{j} t_{N}\right)+r=h\left(t_{N}\right) .
\end{array}\right.
$$

There are $(2 J+1) \times(n+1)$ unknown variables and $N+1$ linear equations in Eq. (7). This system should have a solution based on the least squares fitting method so long as $N \geq(2 J+1) \times(n+1)$.
The statistical properties of the least squares solution can be found in any analysis of variance or regression model text (e.g., Foreman and Henry 1989). According to their theory, the values of $\left\{x^{j}\left(\tilde{t}_{k}\right)\right\}$ and $\left\{y^{j}\left(\tilde{t}_{k}\right)\right\}$, as well 
as $\left\{S_{0}\left(\tilde{t}_{k}\right)\right\}$ for $k=0,1, \ldots, n$ and $j=1,2, \ldots, J$, can be obtained. Then by Eq. (5), amplitudes $\left\{A^{j}\left(\tilde{t}_{k}\right)\right\}$ and phases $\left\{\phi^{j}\left(\tilde{t}_{k}\right)\right\}$ for constituent $j$ on IPs $\tilde{t}_{k}$ can be calculated. Finally, the harmonic parameters at the time index of the whole time domain are obtained using Eq. (3). In another words, the harmonic parameters with temporal variations are obtained.

\section{d. Segmented harmonic analysis}

In this section the segmented harmonic analysis (SHA) algorithm, a method to find evidence of temporal variations in harmonic parameters, is presented as the comparison of EHA. This method is similar to the shortterm harmonic analysis (Jay and Flinchem 1999). Assume we have a time series of data with a total time length of $T$. Actually, CHA treats the whole series as one part. Thus, there is only one amplitude and phase for a specific tidal constituent and one mean flow during the whole time domain. The general idea of SHA is to perform CHA on the subtime series of data. The main process of SHA is presented as follows.

First, the whole time domain is divided into $P$ parts uniformly. As a consequence, the length for every part is $T_{0}=T / P$. The value of $P$ can be assigned according to the length of data. Note that the length $\mathrm{T}_{0}$ should be large enough to ensure the decomposition of a specific number of tidal constituents. Second, CHA is carried out in each part of data. So, we get a constant amplitude $A_{i}$ and phase $\phi_{i}$ of a specific tidal constituent and a mean flow for the $i$ th part of data.

As a result, the time-varying harmonic parameters are described by a series of parameters obtained by SHA. In practice, the SHA results can be interpolated between the central times of each SHA record to obtain time-varying and smoothing harmonic parameters. Jay and Flinchem (1999) have demonstrated, however, that segmented harmonic analysis will sometimes give unstable or erroneous results as the window is stepped through a nonstationary data record (Jay and Kukulka 2003). So, this method will be used only as a reference in this paper.

\section{e. Constituent selection and significance of estimates}

There are more than 146 possible tidal constituents that can be included in the traditional tidal analysis, of which 45 are astronomical in origin (main constituents) and the remaining constituents are shallow water constituents (Foreman 1977), which may be insignificant for internal tides. Moreover, resolving a large number of constituents for a nonstationary signal (e.g., internal tides) is meaningless. Deciding which major constituents should be included in the first stage of a harmonic analysis is not easy (Foreman et al. 2009) and usually begins by considering the so-called Rayleigh criterion
(Godin 1972), which is the minimal resolvable frequency separation of $T^{-1}$ between neighboring constituents in a time series of length $T$. The Rayleigh separation equation can be written as

$$
\left|\sigma_{2}-\sigma_{1}\right|>T^{-1} R
$$

where $\sigma_{1}$ and $\sigma_{2}$ are major constituent frequencies (cycle/unit time), and $R$ is the Rayleigh constant. For harmonic analysis on observational time series, $R$ is commonly set to 1 (Foreman and Henry 1989).

However, Matte et al. (2013) indicated that the Rayleigh criterion cannot be directly used in the nonstationary case because it may be too exclusive for low frequencies or too inclusive for higher frequencies (Jay and Flinchem 1999). Munk and Hasselmann (1964) argued that unlike the pure spectral lines for stationary signals, nonstationarity adds a continuous spectral line to cusp-like-shaped peaks that reflect the intensity to modulate the tides. The width of these cusps reflects the intensity of modulation of the tidal components (Matte et al. 2014). So, even though the close frequencies can be resolved by a Rayleigh criterion based on the length of record (Godin 1972), their overlapping cusps will lead to erroneous estimates of tidal properties (Godin 1999; Colosi and Munk 2006). To solve the dilemma, Matte et al. (2013) defined a new Rayleigh criterion for their tidal-fluvial model. According to the research of Munk and Hasselmann (1964), the width of the cusps formed around the tidal spectral lines reflects the convolution from low-frequency spectrum of the nonlinear forcing.

In this paper we borrow from the work of Matte et al. (2013) and redefine the Rayleigh criterion of harmonic analysis for nonstationary internal tides. For two tidal frequencies $\sigma_{1}$ and $\sigma_{2}$, the minimal allowable frequency separation $\Delta \sigma$ is presented as $\left|\sigma_{2}-\sigma_{1}\right|>\Delta \sigma$,

$$
\left|\sigma_{2}-\sigma_{1}\right|>\max \left(T^{-1} R, \Delta \sigma_{c}\right),
$$

where $T^{-1} R$ is based on the classical harmonic analysis and $\Delta \sigma_{c}$ is the allowable frequency separation for nonstationary signals. The quantity $\Delta \sigma_{c}$ can be determined based on the definition of Munk and Hasselmann (1964):

$$
\frac{\int_{0}^{\Delta \sigma_{c}} P(\sigma) d \sigma}{\int_{0}^{\infty} P(\sigma) d \sigma}=\varepsilon,
$$

where $P(\sigma)$ is the power spectrum of the current data and $\varepsilon$ is a user-defined criterion representing the portion of the total spectrum power. After selecting the constituent, the constituent amplitudes and phases were carefully inspected to ensure that the included 
constituents have a physical meaning. Under the assumption that $\mathrm{CHA}$ accurately represents the average frequency content of the time series, comparisons with the time-averaged tidal harmonics given by $\mathrm{CHA}$ are carried out. Any constituents exhibiting nonphysical characteristics will be eliminated from the analyses.

Closely related to the Rayleigh criterion is the post facto determination of the significance of the estimates. There are various approaches to do this, and the continuous wavelet transform (CWT) method might be an efficient and accurate approach (Jay and Flinchem 1997) to show which constituent is significant in its species. The CWT results mostly provide us the qualitative view of constituent significance. In the quantitative view, the signal-tonoise ratio (SNR), which is the square of the ratio of the tidal amplitude to amplitude error, is often regarded as the indicator of the constituent significance (Pawlowicz et al. 2002; Matte et al. 2013). Since the amplitude and its errors are functions of time in this paper, the SNR should also vary in time. So, the significance of constituents is assessed based on the time-averaged SNR. The modern way to deal with nonstationary mapping on this issue is through resampling techniques, such as the "parametric bootstrap" algorithm (Efron and Tibshirani 1994) used in this paper. The bootstrap technique is a straightforward way to derive estimates of standard errors and confidence intervals for complex estimators of complex parameters of the distribution. Although for most problems it is impossible to know the true confidence interval, the bootstrap technique is asymptotically more accurate than the standard intervals obtained using sample variance and assumptions of normality (DiCiccio and Efron 1996).

The SNR is actually the overall quantitative estimation for each constituent during the time domain. Time-varying quantitative confidence intervals will also be provided as a reference of uncertainties. According to Chavanne et al. (2010), uncertainties are estimated by a bootstrap technique, which is the same as in the SNR calculations. First, the tidal currents estimated by EHA are removed from the original observed time series to obtain the residual currents, or noise. Then, 300 synthetic noise realizations are generated by resampling the time series of residual currents. Third, tidal currents are added back to each noise realization, from which a new estimate of tidal currents is obtained by means of least squares fit. Finally, 95\% confidence intervals on the harmonic parameters are obtained from histograms of the 300 realizations.

\section{Numerical example}

In this section a numerical example is described to demonstrate that EHA can reduce the error of HA and reveal the time-varying amplitudes, phases, and mean flow for the tidal current data. First, we simulate a time series of simple tidal current affected by noise. Then, three HA algorithms-CHA, EHA, and SHA-are applied to the tidal current data. Finally, the analysis results are preliminarily discussed to examine the performances of the three algorithms.

To sufficiently resemble real current data, we first exact a 1-yr time series of current data starts from 0000 UTC 22 October 2010 from the mooring current observations located in the SCS (detailed in next section) as a reference to construct the synthetic tidal signal. Second, traditional harmonic analysis using the T_TIDE package (Pawlowicz et al. 2002) is performed on the series of data to obtain the average values as well as the $95 \%$ confidence interval (CI) estimates of eight tidal constituents' properties and the mean tidal current. The signal-to-noise ratios (SNR) of six significant constituents including $\mathrm{M}_{2}, \mathrm{~K}_{1}, \mathrm{~S}_{2}$, $\mathrm{O}_{1}, \mathrm{~K}_{2}$, and $\mathrm{P}_{1}$ (sorted by SNR) are bigger than 1, indicating they are significant. But according to the Rayleigh criterion, including $\mathrm{K}_{2}$ and $\mathrm{P}_{1}$ will influence the results of $S_{2}$ and $K_{1}$. Besides, the SNR for $K_{2}$ and $P_{1}$ are much smaller to the other constituents. So they are excluded from the numerical experiments. Finally, we construct a synthetic tidal current signal composed of a time-varying nontidal current $U_{0}$ using the four significant constituents; the Gaussian noise $\eta$, where the mean value is $\mu=0 \mathrm{~m} \mathrm{~s}^{-1}$; and the variance $\sigma=0.01 \mathrm{~m}^{2} \mathrm{~s}^{-2}$. All eight components are described by time-varying amplitudes and phases. With a sample interval of $\Delta t$, the tidal current $U$ at time $t_{i}=t_{0}+i \Delta t$ can be described as follows:

$$
\begin{aligned}
U\left(t_{i}\right)= & U_{0}\left(t_{i}\right)+\eta\left(t_{i}\right) \\
& +\sum_{m=1}^{4} A_{m}\left(t_{i}\right) \cdot \cos \left[2 \pi \theta_{m} i \Delta t-\phi_{m}\left(t_{i}\right)\right],
\end{aligned}
$$

where the temporal variational $A_{m}, \phi_{m}$, and $U_{0}$ are given by the following equations:

$$
\begin{aligned}
A_{\mathrm{M} 2}\left(t_{i}\right) & =0.11+0.01 \times \sin [2 F(i)], \\
\phi_{\mathrm{M} 2}\left(t_{i}\right) & =359.27+5.68 \times \cos [F(i)], \\
A_{\mathrm{S} 2}\left(t_{i}\right) & =0.04+0.01 \times \cos [2 F(i)], \\
\phi_{\mathrm{S} 2}\left(t_{i}\right) & =74.91+16.89 \times \cos [F(i)], \\
A_{\mathrm{O} 1}\left(t_{i}\right) & =0.04+0.02 \times \sin [F(i)], \\
\phi_{\mathrm{O} 1}\left(t_{i}\right) & =267.82+21.89 \times \cos [2 F(i)], \\
A_{\mathrm{K} 1}\left(t_{i}\right) & =0.09+0.02 \times \sin [F(i)], \\
\phi_{\mathrm{K} 1}\left(t_{i}\right) & =14.94+10.14 \times \cos [2 F(i)], \\
U_{0}\left(t_{i}\right) & =-0.0426+0.02 \times \cos [2 F(i)],
\end{aligned}
$$

where $F(i)=\pi i \cdot \Delta t / 8760$. 

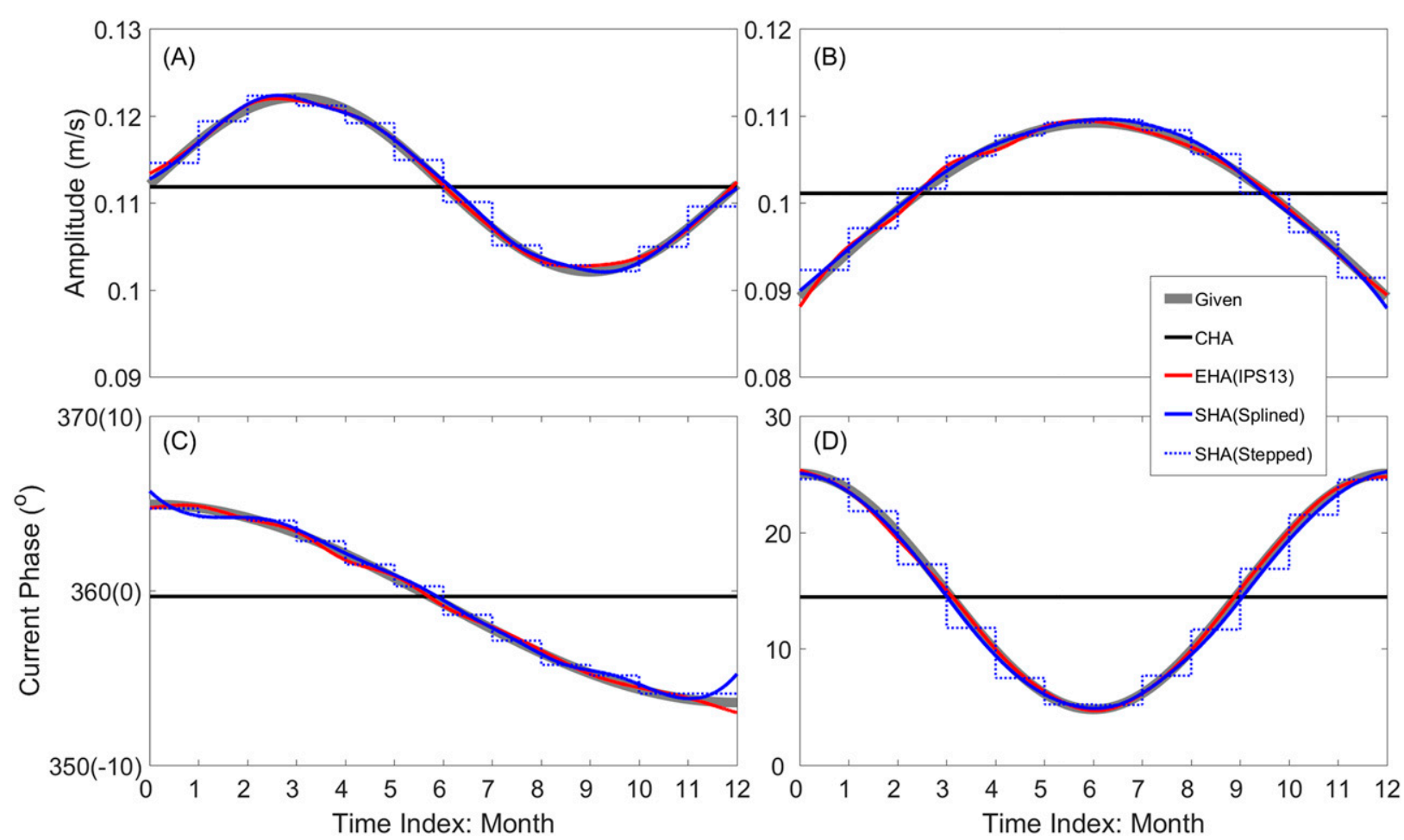

FIG. 2. Given values and HA results of current amplitudes (a) $K_{1}$ and (b) $M_{2}$, and (c),(d) their respective phases. The horizontal gray lines are the given values; the black line is the CHA, the red is the EHA, the solid blue is the SHA (Splined), and the dotted-stepped blue is the SHA (Stepped).

Three HA algorithms-CHA, EHA, and SHA-are applied to the artificial current data. The constituent sets used for CHA, EHA, and SHA, which are $\mathrm{M}_{2}, \mathrm{~S}_{2}, \mathrm{O}_{1}$, and $\mathrm{K}_{1}$, are exactly the same for comparison. Note that the time-varying mean flow will also be resolved. Four different EHAs that employ different IPSs are applied to examine the accuracy and feasibility of this algorithm. As for the SHA, a total of 12-subtime series of data are selected by step 1 in section 2 ; t the time length for each subtime series is one month. The phases are reduced to Greenwich values, and no nodal modulations are included in the three algorithms.

All harmonic results, including the current amplitudes, phases, and the time-varying mean flow (not shown in figures), with different methods, are calculated, but the results from only $\mathrm{M}_{2}$ and $\mathrm{K}_{1}$ are shown in Fig. 2 as examples. Harmonic results for all the selected constituents are provided in the appendix for readers who are interested. From now on, we denote the IPS $n$ as the IPS that concludes a total number of $n$ in EHA. For clarity, we present only the results of IPS13 of the EHA algorithm in the figure. On the one hand, these results clearly show the deficiency of CHA. It is obvious that the amplitude and phase constants computed by $\mathrm{CHA}$ fail to reveal the temporal variations of harmonic parameters for the two given tidal constituents. On the other hand, the harmonic results for SHA marked step changes with time, which is obviously not a good choice to reveal the continuous and smooth temporal variations of harmonic parameters. To acquire time-varying harmonic parameters, the harmonic results for SHA are interpolated with cubic splines between the central times of each SHA record (splined SHA). The mean difference between EHA and splined SHA lies in the fact that the former is using the data from the entire record to determine the harmonic parameters at any particular point with the spline coupling included. In another words, the time length to determine the harmonic parameters for SHA is the length of each segment (one month for this issue), while the time length for EHA is the length of the whole record (12 months for this issue). The root-meansquare errors (RMSEs) of the harmonic parameters, including the current amplitudes $(\delta A)$, phases $(\delta \phi)$, and mean flow $\left(\delta S_{0}\right)$, as well as those between the tidal predictions (TP) and the simulated data (SD), which are an indicator of model-data misfit for all algorithms ( $\delta$ Data), are calculated and listed in Table 1.

Based on the statistics in Table 1 and the curves in Fig. 2, the performances of the three HA algorithms are evaluated in the following paragraph. In terms of the RMSE for the six tidal current amplitudes $(\delta A)$, phases $(\delta \phi)$, and mean flow $\left(\delta S_{0}\right)$, the splined SHA and EHA 
TABLE 1. Estimated RMSE for three HA algorithms $\left(\mathrm{m} \mathrm{s}^{-1}\right)$.

\begin{tabular}{lcccccc}
\hline \multicolumn{1}{c}{ Method } & CHA & SHA & EHA (IPS5) & EHA (IPS9) & EHA (IPS13) & EHA (IPS16) \\
\hline$\delta A\left(\mathrm{~m} \mathrm{~s}^{-1}\right)$ & $7.5375 \times 10^{-3}$ & $4.2762 \times 10^{-4}$ & $3.1251 \times 10^{-4}$ & $1.6911 \times 10^{-4}$ & $2.4813 \times 10^{-4}$ & $3.1037 \times 10^{-4}$ \\
$\delta \phi\left(^{\circ}\right)$ & 14.5872 & 0.6607 & 0.5812 & 0.0481 & 0.0166 & 0.0108 \\
$\delta S_{0}\left(\mathrm{~m} \mathrm{~s}^{-1}\right)$ & $1.4143 \times 10^{-2}$ & $3.1636 \times 10^{-4}$ & $7.6191 \times 10^{-4}$ & $1.4031 \times 10^{-4}$ & $1.4208 \times 10^{-4}$ & $1.6839 \times 10^{-2}$ \\
$\left.\delta \mathrm{Data}^{-1} \mathrm{~m} \mathrm{~s}^{-1}\right)$ & $9.0291 \times 10^{-2}$ & $8.7061 \times 10^{-2}$ & $3.3853 \times 10^{-2}$ & $3.3370 \times 10^{-2}$ & $3.3364 \times 10^{-2}$ & $3.3359 \times 10^{-2}$ \\
\hline
\end{tabular}

are competitive, and both of them have much better performances than CHA. Moreover, the errors vary among experiments employing EHA. The values of $\delta$ Data for SHA and EHA are smaller than that for CHA. All the evidence indicates the significant advantages of SHA and EHA over CHA.

This simple example clearly demonstrates that both SHA and EHA (employing different IPSs) have the ability to improve tidal current estimates for a synthetic signal, compared with CHA. The harmonic parameters of EHA and the splined results of SHA are smoothed; so, they are more reasonable in physics. However, Figs. 3-5 do not inspire a lot of confidence in the smoothness of the data. This is mainly because of two reasons. First, any form of HA provides average pictures of the harmonic parameters rather than instantaneous results. Second, there is an inevitable trade-off between time and frequency resolution when we select the candidate constituents to be analyzed, and there is no single answer when the data are nonstationary. Moreover, different IPSs in EHA may result in different harmonic results. To investigate this issue, a variety of EHAs with different IPSs are performed on the same artificial current data. When the number of IPs exceeds 21 , the harmonic results begin to reveal unnecessary oscillations compared to the prescribed curves (for figures not shown in the paper; please refer to the supplementary file, file JTECH-D-16-0239s1), and the RMSEs for amplitudes and phases begin to rise. This phenomenon is easy to understand from the introductions in section $2 c(2)$, because the increase in the IP number means the increase in the undetermined parameters in the harmonic analysis. Thus, it will lead to more uncertainty to solve the harmonic linear Eqs. (6). Moreover, in the area of computational statistics, a lot of effort was put forward to prevent overfitting of curves by reducing the number of knots required to represent the original curve with a given degree of accuracy (Qamar 1993). So, it is not a good decision to use an abundant number of IPs in EHA mainly for two reasons. First, the increase in the number of IPs fails to result in a decrease in the error estimates when the number of IPs exceeds 13. In other words, it is inefficient to use an excessive number of IPs in the method of EHA. Second, the physical meaning of IPS has been clearly clarified in section $2 \mathrm{~b}$. Thus, redundant IPs applied in EHA mean dramatic temporal changes for estimated tidal current amplitudes and phases all the time, which is inconsistent with the

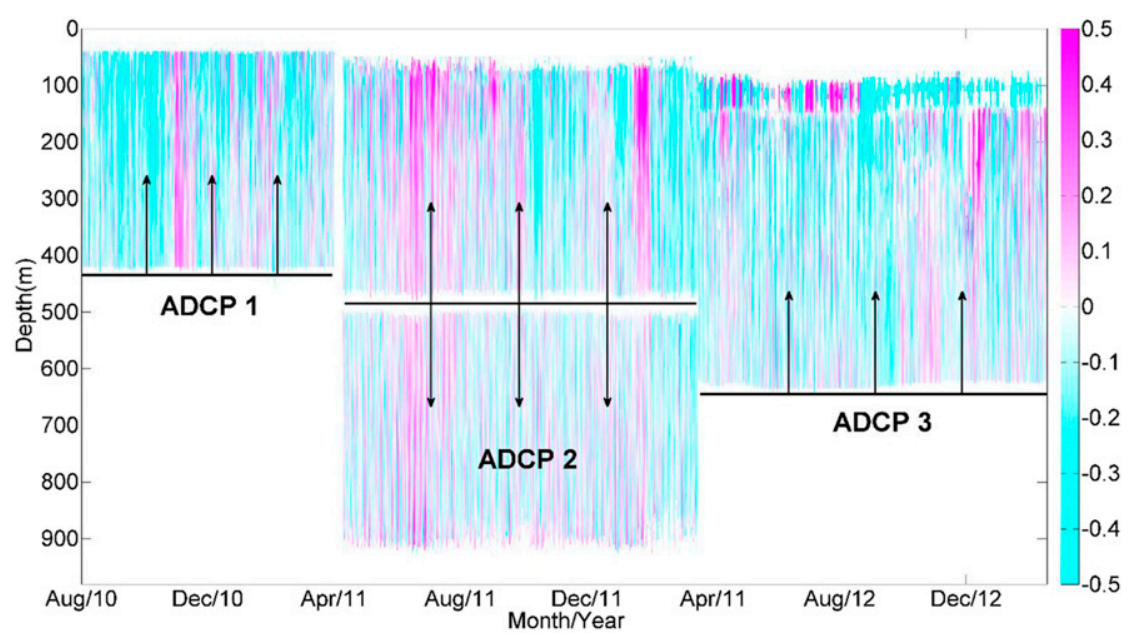

FIG. 3. Original meridional current data obtained by three ADCPs. The position and working time for ADCPs are horizontal black lines. The direction of the vertical black arrows shows the type of ADCP (upward arrows for the uplooking ADCP and double arrows for the side-looking ADCP). The color scale on the right starts at 0.5 at the top and extends to -0.5 at the bottom in $0.1 \mathrm{~m} \mathrm{~s}^{-1}$ increments. 

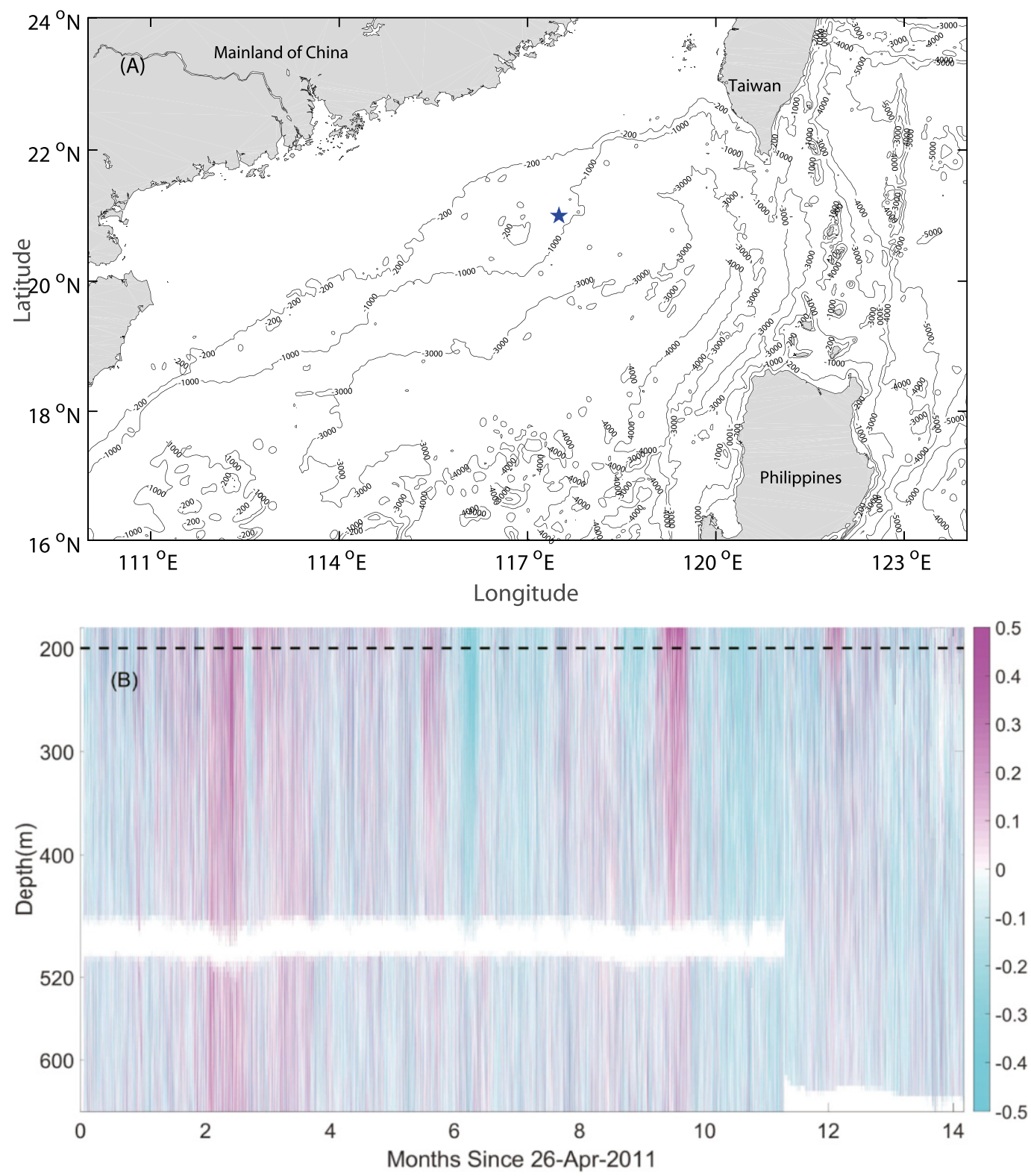

FIG. 4. (a) Map of the northeastern portion of the SCS and position of ADCP mooring site (blue star near $21^{\circ} \mathrm{N}$, $\left.118^{\circ} \mathrm{E}\right)$. (b) Hourly zonal component $u\left(\mathrm{~m} \mathrm{~s}^{-1}\right)$ of the observed mooring current data from 26 Apr 2011 to $24 \mathrm{Jun}$ 2012. The dashed line indicates the 200-m depth of the current data used in this section.

physical movements of tidal currents. A notable fact in the current data and the wavelet spectrum is that internal tides do change abruptly. However, we are limited in our ability to resolve this by the Heisenberg principle (Heisenberg 1927).

\section{Application to the mooring observations}

In the following section, the performances of the three HA algorithms-CHA, SHA and EHA-are compared when applied to the mooring current observations in the
SCS. A 14-month (26 April 2011-24 June 2012) time series of current data (original data shown in Fig. 3) is picked out from $2.5 \mathrm{yr}$ of current data obtained by acoustic Doppler current profilers (ADCP). The mooring was deployed during the South China Sea Internal Wave Experiment (Zhang et al. 2013; Guan et al. 2014) and are part of the South China Sea Moored Observation Network of China. The data before 2200 UTC 17 April 2011 were obtained from a $75-\mathrm{kHz}$ uplooking ADCP at $435 \mathrm{~m}$, data from 0000 UTC 30 April 2011 to 0600 UTC 30 March 2012 were obtained from a 


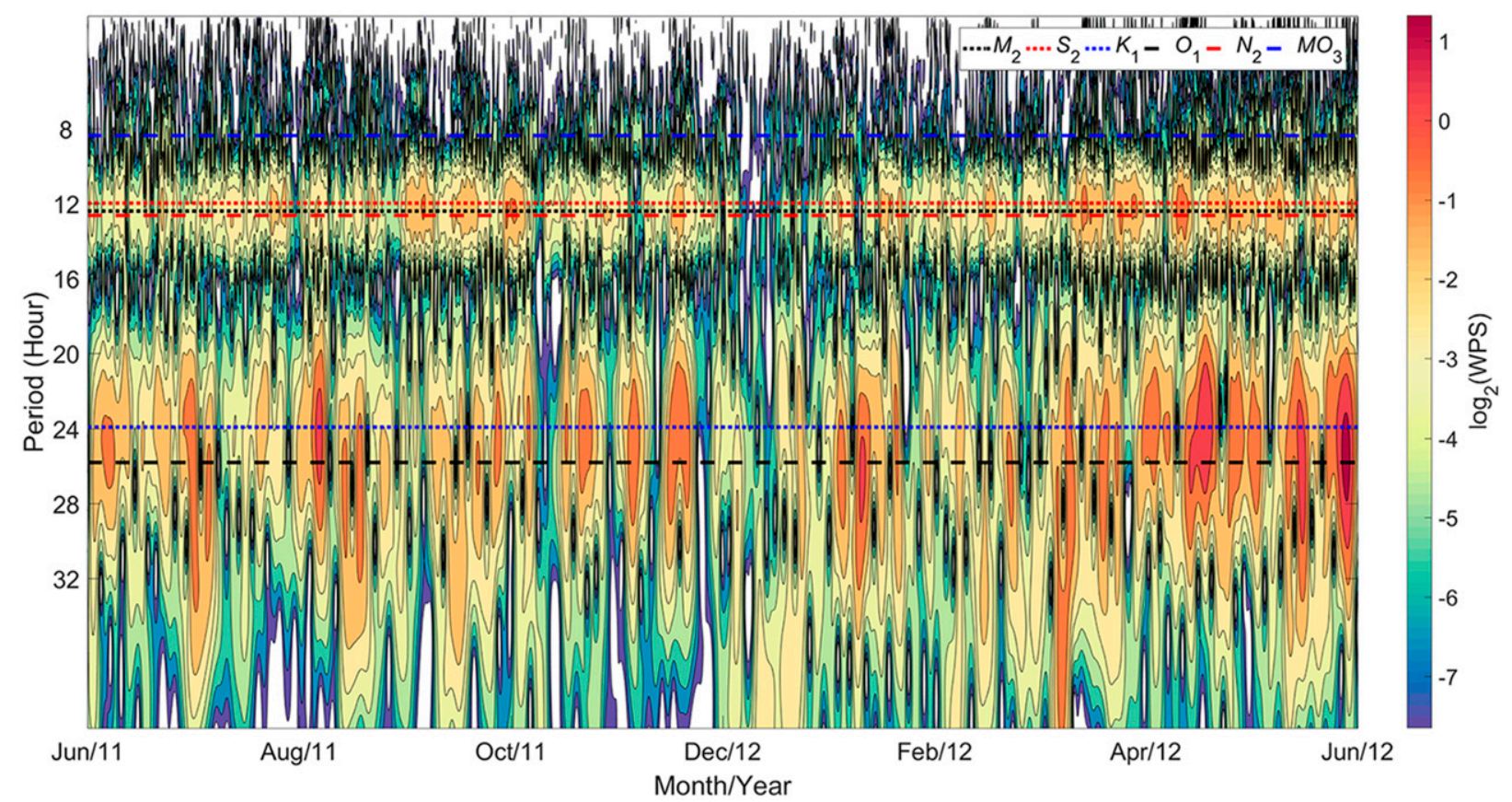

FIG. 5. The local wavelet power spectrum (color shading) of the current data time series using the Morlet wavelet. The colored lines represent the following tidal components: $\mathrm{M}_{2}$ (black dashed), $\mathrm{S}_{2}$ (red dashed), $\mathrm{K}_{1}$ (blue dashed), $\mathrm{O}_{1}$ (solid black), $\mathrm{N}_{2}$ (solid red), and $\mathrm{MO}_{3}$ (solid blue).

$75-\mathrm{kHz}$ side-looking ADCP at $485 \mathrm{~m}$, and data after 1300 UTC were obtained from a $75-\mathrm{kHz}$ uplooking ADCP located at $630 \mathrm{~m}$. As is shown in Fig. 4a, the mooring site was located at $21^{\circ} 07^{\prime} \mathrm{N}, 117^{\circ} 53^{\prime} \mathrm{E}$. The water depth at the mooring site is $968 \mathrm{~m}$, and the depth of the available current data measured by the two ADCPs ranged from the sea surface to 940 and $640 \mathrm{~m}$ with a vertical interval of $5 \mathrm{~m}$, respectively. Current measurements were recorded with a precision of $1 \times 10^{-4} \mathrm{~m} \mathrm{~s}^{-1}$ and a time interval of $1 \mathrm{~h}$. The current data located at the $200 \mathrm{~m}$ are selected to exclude not-a-number ( $\mathrm{NaN}$ ) values. Because the main purpose of this section is to examine the capability and feasibility of EHA, only the meridional component is employed. Figure $4 \mathrm{~b}$ shows the hourly component $u$, which will be used to examine the capability of the three HA algorithms mentioned above.

We start with applying the three HA algorithms to the 1-yr mooring current data from 26 April 2011 to 25 April 2012 at a depth of $200 \mathrm{~m}$. As introduced in section 2, the whole process for EHA, including constituent selection and significant analyses, can be executed in the following steps:

Step 1. Calculate the minimal allowable frequency separation $\Delta \sigma$ based on the length of record and the power spectra of the three HA algorithmsCHA, EHA, and SHA - are applied to time series $(\varepsilon=0.3$; see section $2 \mathrm{e}$ for the definition).
Step 2. Apply CHA to the time series in order to evaluate the time-averaged amplitudes and phases for the constituents. Include constituents whose frequencies are under the limit of $\Delta \sigma$. Exclude those constituents with nonphysical characteristics.

Step 3. Apply EHA to the time series and obtain harmonic parameters with time variations.

Step 4. Perform CWT with the wavelet package in MATLAB (Torrence and Compo 1998) to the time series to acquire local wavelet power spectra throughout the sample time.

Step 5. Calculate the SNR as a function of time using the parametric bootstrap algorithm (Chavanne et al. 2010). The time-averaged SNR can be treated as the overall quantitative indicator for the significance of constituents during the time domain. Moreover, time-varying quantitative $95 \%$ confidence intervals are also calculated by the parametric bootstrap algorithm.

The minimum allowable frequency separation based on the power spectra in step 1 of the current time series is 0.0356 cycles per day (cpd), which means that only constituents separated by about 28 days per cycle would be selected. The result of this criterion argues that including more constituents within each "monthly" group would be inconsistent with the variability in the signal. So, the time length for each segment (one month) in 


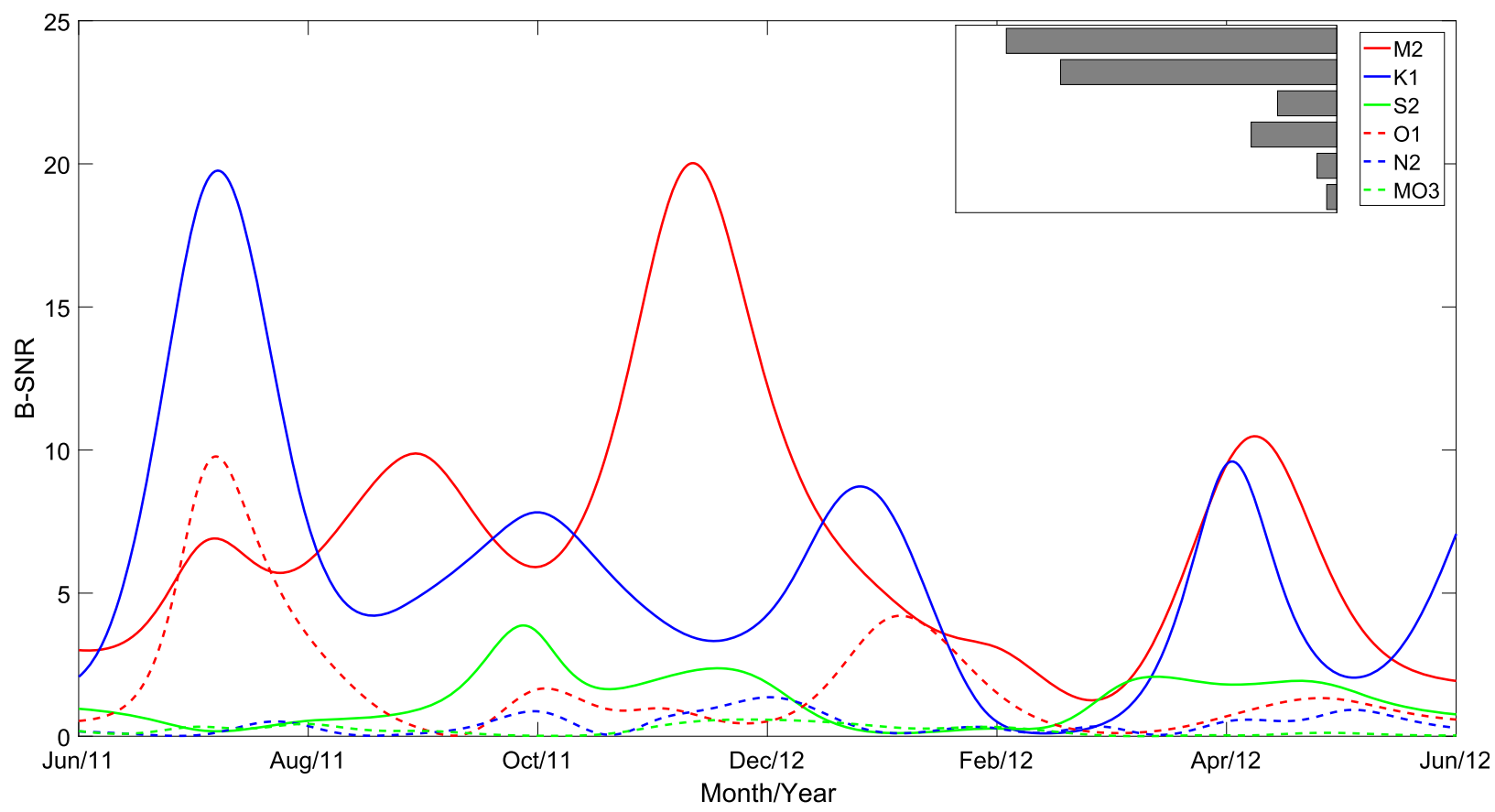

FIG. 6. Time variations and (inset) time-averaged values of B-SNR for six constituents: $\mathrm{M}_{2}$ (solid red), $\mathrm{K}_{1}$ (solid black), $\mathrm{S}_{2}$ (solid green), $\mathrm{O}_{1}$ (dashed red), $\mathrm{N}_{2}$ (dashed blue), and $\mathrm{MO}_{3}$ (dashed green).

SHA is reasonable for the current data. With regard to the constituent selection, we first choose a total of 13 candidates out of the significant constituents based on the SNR of the CHA results. Then, the candidates are filtered with $\Delta \sigma$. Finally, six constituents $-\mathrm{M}_{2}, \mathrm{~S}_{2}, \mathrm{~K}_{1}$, $\mathrm{O}_{1}, \mathrm{~N}_{2}$, and $\mathrm{MO}_{3}$-along with a time-varying mean flow are selected to be determined in the EHA. At the same time, these constituents are also used in CHA and SHA for consistency. The time length of each segment part in SHA is one month, and the harmonic results from SHA are interpolated with cubic splines in this section. Note that among the eight principal constituents, $\mathrm{P}_{1}, \mathrm{Q}_{1}$, and $\mathrm{K}_{2}$ failed to pass the two barriers. The reason is either it is too insignificant throughout the time interval to be selected $\left(\mathrm{Q}_{1}\right.$ and $\left.\mathrm{P}_{1}\right)$ or it fails to pass the examination of $\Delta \sigma\left(\mathrm{K}_{2}\right)$. While investigating internal tides with mooring observations around this area, most researchers pay attention to the four principal constituents, $\mathrm{M}_{2}, \mathrm{~S}_{2}, \mathrm{~K}_{1}$, and $\mathrm{O}_{1}$ (J. Zhang et al. 2011; Lee et al. 2012; Guo et al. 2012; Xu et al. 2013; Gao et al. 2015; Gao et al. 2016), and our result are consistent with previous findings.

Figure 5 gives details about the analysis of the wavelet power spectrum using the Morlet wavelet. Please note that the minimum plotting level in the figure is 0.005 , which means a wavelet power smaller than 0.005 will not be plotted. As can be clearly seen in the figure, most of the power is concentrated within the diurnal band around $12 \mathrm{~h}$ and the semidiurnal band around $24 \mathrm{~h}$.
Moreover, overtides between the diurnal and semidiurnal constituents, such as $\mathrm{MO}_{3}$, can be found near the third diurnal band. This is mainly because of the nonlinear distortions of the major astronomical tidal constituents. More solid evidences will be provided in the spectra (Fig. 11). With wavelet analysis, one can see variations in the frequency of occurrence and amplitude of the current. The wavelet power spectrum reveals quasi-periodic oscillation because of the modulation of tidal forcing. Moreover, this agrees with other studies (Z. Zhang et al. 2011; Lee et al. 2012; Guo et al. 2012; Xu et al. 2013; Gao et al. 2015; Gao et al. 2016) and gives confidence in the constituent selection.

As introduced in section 2e, the so-called colored bootstrap analysis (Pawlowicz et al. 2002) is applied to determine the significance. Figure 6 demonstrates the time variations and the time-averaged values of SNRs obtained by bootstrap analysis (B-SNRs) for six constituents. Note that because of the stochastic nature of the bootstrap procedure, values of B-SNRs may change slightly in repeated analyses. Compared with the results from CHA, the top four significant constituents are exactly the same. However, there exist prominent time variations among the B-SNRs of the six constituents. The $\mathrm{M}_{2}$ and $\mathrm{K}_{1}$ constituents are much more significant than others are most of the time. On the contrary, values of B-SNR for $\mathrm{MO}_{3}$ ramble at a low level for the majority of the time. 

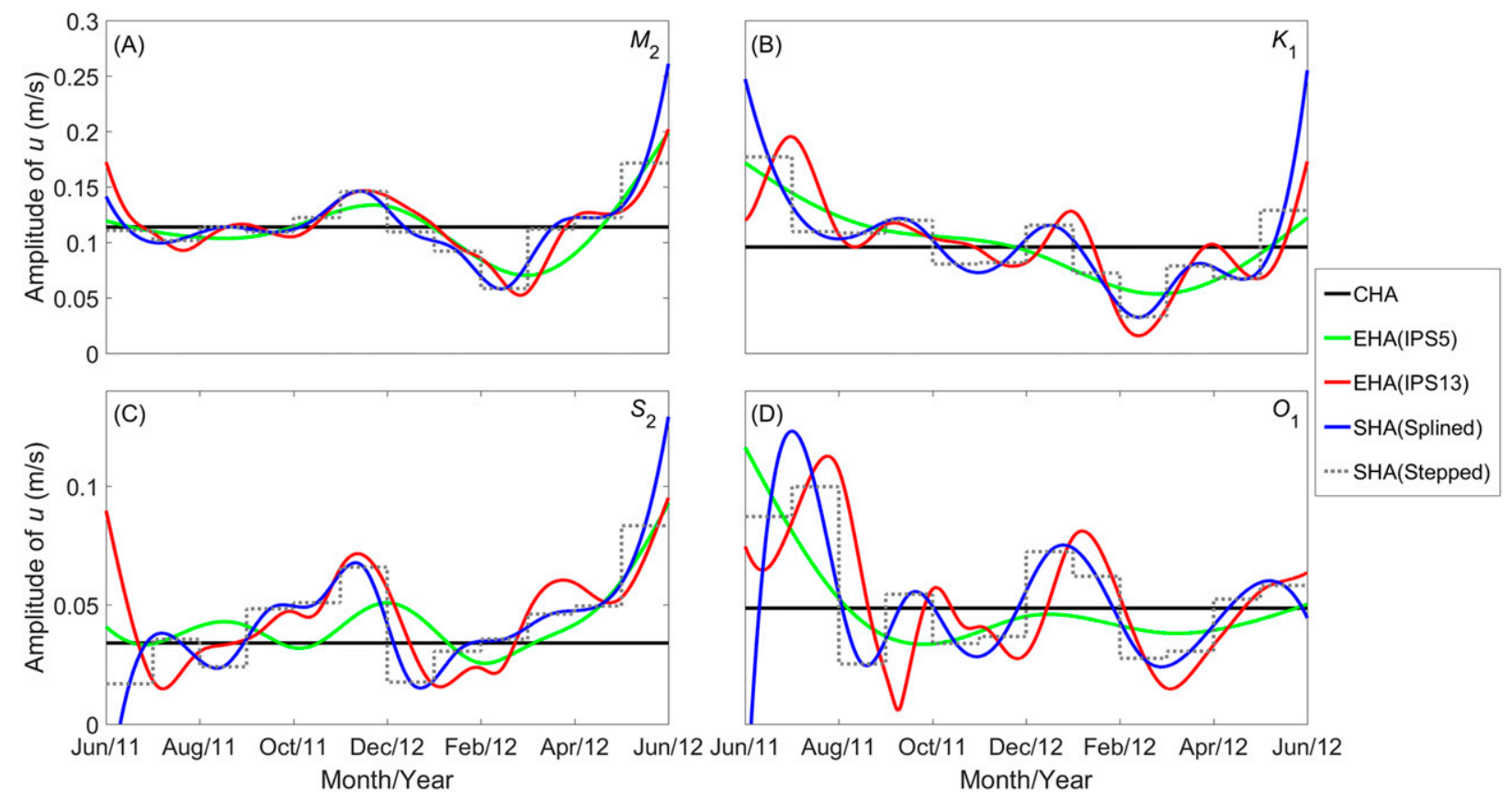

FIG. 7. Results of the time-varying zonal current amplitudes obtained by three HA algorithms at 200-m depth. The four principal tidal constituents are (a) $\mathrm{M}_{2}$, (b) $\mathrm{K}_{1}$, (c) $\mathrm{S}_{2}$, and (d) $\mathrm{O}_{1}$ : CHA (solid black), EHA (IPS5) (green), EHA (IPS13) (red), SHA (Spined) (solid blue), and SHA (Stepped) (dashed blue). Note that "SHA (Stepped)" indicates that the time length of each segment part is one month.

Figure 7 shows the time-varying current amplitudes of four principal tidal constituents- $\mathrm{M}_{2}, \mathrm{~K}_{1}, \mathrm{~S}_{2}$, and $\mathrm{O}_{1}-$ obtained by the three HA algorithms. Note that four IPSs in EHA are applied in this case but only the results of IPS5 and IPS13 are illustrated for clarity. The phases are reduced to Greenwich values, and no nodal modulations are included in the three algorithms. The annualaveraged current amplitudes are obtained by CHA (black lines in Fig. 7). Generally, the amplitudes of $\mathrm{M}_{2}$ and $\mathrm{K}_{1}$ are larger than those of $\mathrm{S}_{2}$ and $\mathrm{O}_{1}$. And as for the harmonic results obtained from SHA and EHA, it should be noted that the seasonal variabilities of the current amplitudes of the four principal tidal constituents are significant. Moreover, the overall trends of the current amplitudes for EHA and SHA are consistent. Taking Fig. $7 \mathrm{~b}$ for instance, the current amplitude for $K_{1}$ is relatively small in winter and larger in summer, which is consistent with the analysis results by $\mathrm{Xu}$ et al. (2013). The phase results and time-varying mean flow are presented in the appendix. As introduced in section $2 \mathrm{e}$, the uncertainties are estimated by the bootstrap technique (Chavanne et al. 2010), and the $95 \%$ confidence intervals for all the harmonic parameters are calculated as quantitative estimations for all constituents. The $95 \%$ confidence intervals for the zonal current amplitudes of the four principal constituents obtained by EHA with IPS13 are provided in the appendix as an example. Readers who may be interested in this issue are referred to the supplementary file for the other results. The confidence intervals at both ends of the harmonic parameters are larger than those in the middle of the time series. This is mainly caused by the interpolations of cubic splines. Thus, an important note for users is that the harmonic results at both ends of the sample time should be used with great care. More evidence of this issue will be presented in the following discussions. The time-varying phases of the four constituents and the mean flow calculated by different algorithms are also presented in the appendix (see Figs. A3, A4). The overall variations in the results from all algorithms show good consistency with each other, which exhibit clear seasonal variations. To compare the harmonic results with different algorithms in detail, the ranges of the current amplitudes and $\delta$ Data are calculated and listed in Table 2. CHA does not have the ability to obtain time-varying current amplitudes, so variations of the four principal constituents are all zero. As for the results of the other two algorithms, the $\mathrm{K}_{1}$ component has the greatest variation, whereas $S_{2}$ has the smallest. Various IPSs in EHA result in apparent differences in the ranges of the current amplitudes, which is as expected. On the other hand, the value of $\delta$ Data varies among the algorithms. Compared with CHA, the application of SHA and EHA reduces the values of $\delta$ Data. With the increase of IPs used in EHA, $\delta$ Data descends slightly from 0.1481 to $0.1402 \mathrm{~m} \mathrm{~s}^{-1}$ and 
TABLE 2. Ranges of estimated current amplitudes for all HA methods and the root-mean-square model-data misfits ( $\mathrm{m} \mathrm{s}^{-1}$ ).

\begin{tabular}{|c|c|c|c|c|c|c|}
\hline Method & CHA & SHA $(12 *)$ & EHA (IPS5) & EHA (IPS9) & EHA (IPS13) & EHA (IPS16) \\
\hline RangeM2 & 0 & 0.2030 & 0.1270 & 0.0836 & 0.1102 & 0.1307 \\
\hline RangeK1 & 0 & 0.2228 & 0.1414 & 0.0928 & 0.1639 & 0.1609 \\
\hline RangeS2 & 0 & 0.1290 & 0.0658 & 0.0619 & 0.0728 & 0.0723 \\
\hline RangeO1 & 0 & 0.1229 & 0.0941 & 0.0690 & 0.1059 & 0.1160 \\
\hline$\delta$ Data & 0.2328 & 0.2224 & 0.1481 & 0.1453 & 0.1423 & 0.1402 \\
\hline
\end{tabular}

* Note that there are 12 segments used in SHA, which is consistent with EHA (IPS13).

the decline rate becomes smaller (more evidence can be found in Fig. 8). Although the magnitudes of $\delta$ Data for SHA and EHA are the same with that for CHA, the application of SHA and EHA acquires current amplitudes with temporal variations. Specifically, with the aid of EHA, the current amplitudes are presented with continuous temporal variations. Additionally, SHA will be more limited to select candidate constituents, as the record length for this algorithm (one month in this case) is much smaller than that in EHA ( $1 \mathrm{yr}$ in this case). It is true that all the three algorithms are limited by noise and nonstationary behavior, but EHA is the best choice to determine current amplitudes with temporal variations. Nevertheless, the performances of EHA with different IPSs vary with respect to ranges of the current amplitudes, whereas the values of $\delta$ Data are of the same magnitude (Fig. 8). As a consequence, how to choose an optimal IPS for EHA is still a challenge.

In the following paragraphs, the capability of EHA is evaluated and the optimal IPS for EHA is selected. In the catalog of smoothing splines, the problem of "knot selection" helps determine the optimal number and locations of knots. Qamar (1993) argued that any method used to determine the knots for cubic spline fitting should guarantee the small error between the fitted curve and the original curve, as well as the retainment of the main features of the original curve with a small number of knots. However, the automatic knot selection for EHA is so complicated that it is beyond the scope of this paper. So, we would just leave it to the next paper.
Nevertheless, we will examine the two aspects to determine the optimal IPS manually under the guidance of Qamar (1993). The first one is the value of $\delta$ Data, which defines the time-averaged difference between observations and the reconstructed time series. The second one is the averaged standard deviation for different time offsets to evaluate the capability of EHA. Figure 8 presents the values of $\delta$ Data with respect to the number of IPs. Compared to the results of CHA and splined SHA, the values of $\delta$ Data obtained with EHA are relatively small. The descent rate is getting smaller when the number of IPs increases. Besides, the results of the numerical examples in section 3 indicate that the abundant number of IPs in EHA may lead to unnecessary oscillations compared to the real temporal variations. As a result, the selection of IPS should be careful enough to avoid overfitting of the harmonic parameters. In this paper we present a method to evaluate the capability of EHA quantitatively. Based on the analysis given above, the optimal EHA should reveal temporal trends for the current amplitudes of all tidal constituents. In other words, harmonic results from the optimal EHA should be consistent for all selected time series with the same time window. Take the 14 months of current data at the depth of $200 \mathrm{~m}$ as an example. As shown in Fig. 9, three subsets of current data are cut out, each containing 12 months of current data. The start month for series 1 (TS1), series 2 (TS2), and series 3 (TS3) are the first, second, and third month of the 14 months of data, respectively. The optimal IPS for

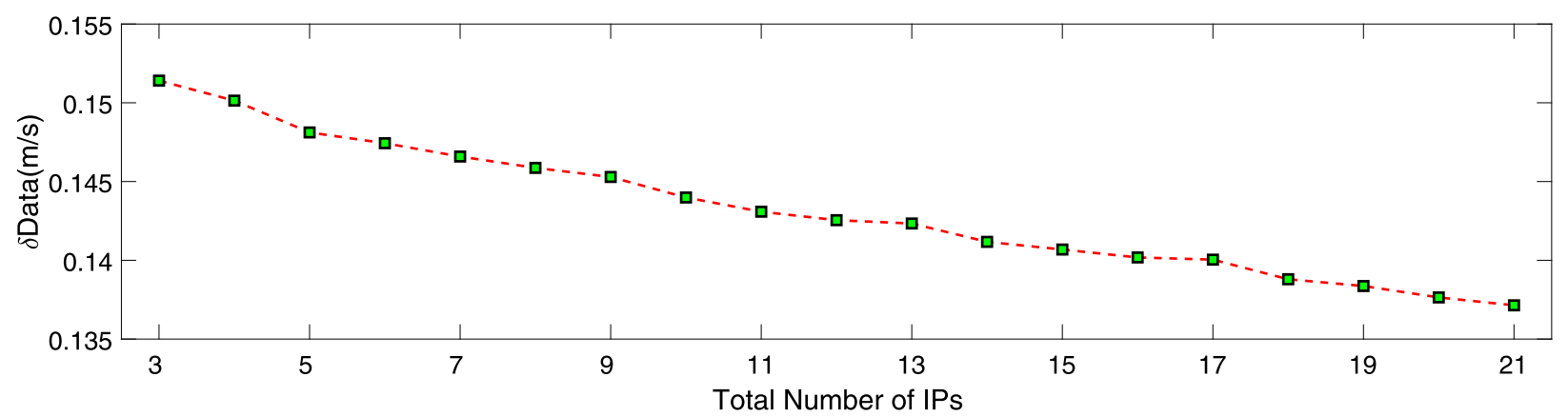

FIG. 8. Values of $\delta$ Data $\left(\mathrm{m} \mathrm{s}^{-1}\right)$ with respect to the number of IPs. 


\begin{tabular}{|c|c|c|c|c|c|c|c|c|c|c|c|c|c|c|}
\hline$T$ & 1 & & & & & & & & & & & 1 & T & \multirow{3}{*}{$\begin{array}{l}\text { Series } 1 \\
\text { Series } 2\end{array}$} \\
\hline \multirow[t]{3}{*}{ A1 } & A2 & A3 & A4 & A5 & A6 & A7 & $A 8$ & A9 & A10 & A11 & $\mathrm{A} 12$ & & & \\
\hline & \multirow[t]{2}{*}{ B1 } & B2 & B3 & B4 & B5 & B6 & B7 & B8 & B9 & B10 & B11 & B12 & & \\
\hline & & C1 & $\mathrm{C} 2$ & $\mathrm{C} 3$ & $\mathrm{C} 4$ & C5 & $\mathrm{C} 6$ & C7 & $\mathrm{C} 8$ & $\mathrm{Cg}$ & C10 & C11 & $\mathrm{C} 12$ & Series 3 \\
\hline 1 & 2 & 3 & 4 & 5 & 6 & ${ }^{7} \mathrm{M}$ & $\begin{array}{c}8 \\
\text { nth }\end{array}$ & $\begin{array}{r}9 \\
\text { ex }\end{array}$ & 10 & 11 & 12 & 13 & 14 & \\
\hline
\end{tabular}

FIG. 9. Sketch illustrating the method to determine the optimal EHA. The shadow region indicates the overlap time of the three series.

EHA will be chosen with the following steps. First, EHA with a specific IPS is applied to each time series and the current amplitudes during the corresponding subset are obtained. Second, the consistency among the three groups of obtained current amplitudes during the overlap time is calculated using

$$
S=\sum_{t=1}^{T} \sqrt{\frac{1}{3} \sum_{i=1}^{3}\left(A_{i, t}-\frac{1}{3} \sum_{i=1}^{3} A_{i, t}\right)^{2}},
$$

where $T$ is the amount of sample points in the shadow region and $A_{i, t}$ refers to the value of current amplitude for series $i$ on the $t$ th sample moment. Actually, the value of $S$ is the averaged standard deviation (ASD) for the three time series inside the shadow region. The smaller the ASD is, the more consistent the harmonic results will be with each other. Finally, by comparing values of $S$ obtained by different EHAs, the capability of EHA is assessed and the optimal EHA can be selected.

Figure 10 shows consistencies among the $\mathrm{M}_{2}$ current amplitudes at the depth of $200 \mathrm{~m}$ obtained by different IPSs applied in EHA. Moreover, we present the ASDs of different IPSs for the four constituents in Table 3. The values of ASD vary with different IPS and different constituents. But the results of IPS13 are interestingly the smallest among the four constituents. Take the results of $\mathrm{M}_{2}$ as an example. The ASD for IPS13 is 0.0013 , which is the smallest among the six experiments. It is $86.22 \%$ smaller than that for IPS5, $66.49 \%$ smaller than
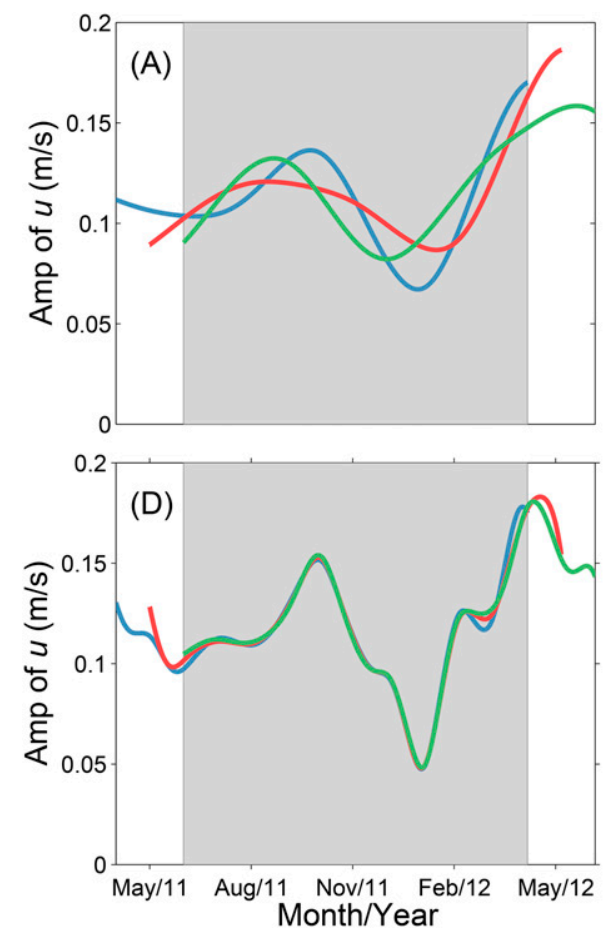
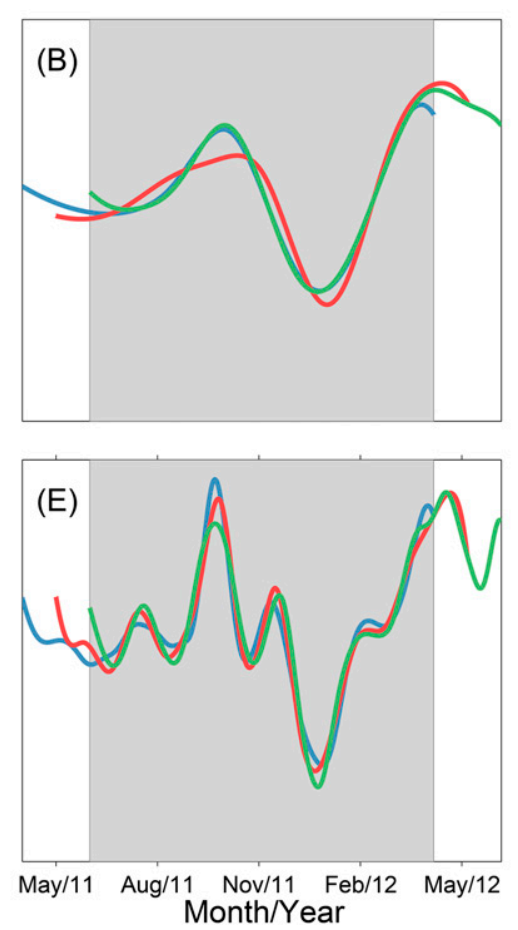
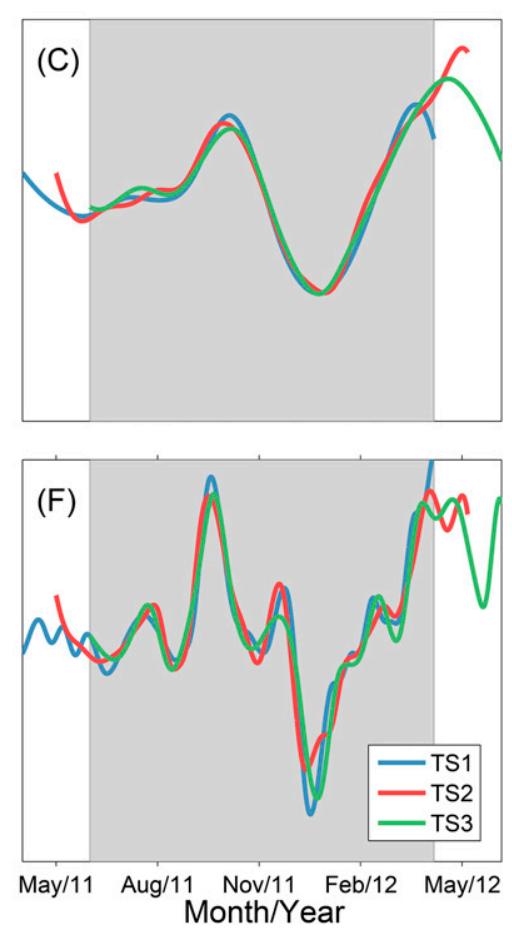

FIG. 10. Comparison of harmonic results for the $\mathrm{M}_{2}$ constituent at 200-m depth with different EHAs. There are (a) 5, (b) 7, (c) 9, (d) 13, (e) 16, and (f) 21 IPs in the experiments. The TS1 (blue line), TS2 (red line) and TS3 (green line) refer to series 1, 2 and 3, respectively. 
TABLE 3. ASDs of different IPSs for the four constituents.

\begin{tabular}{lcccccc}
\hline \hline & IPS5 & IPS7 & IPS9 & IPS13 & IPS16 & IPS21 \\
\hline $\mathrm{M}_{2}$ & 0.0095 & 0.0039 & 0.0026 & 0.0013 & 0.0047 & 0.0058 \\
$\mathrm{~K}_{1}$ & 0.0065 & 0.0053 & 0.0118 & 0.0023 & 0.0085 & 0.0097 \\
$\mathrm{~S}_{2}$ & 0.0076 & 0.0022 & 0.0041 & 0.0011 & 0.0037 & 0.0045 \\
$\mathrm{O}_{1}$ & 0.0085 & 0.0035 & 0.0060 & 0.0013 & 0.0048 & 0.0065 \\
\hline
\end{tabular}

that for IPS7, 50.35\% smaller than that for IPS9, 72.34\% smaller than that for IPS16, and $77.28 \%$ smaller than that for IPS21. This distinct advantage convinces us that EHA with IPS13 can be chosen as the optimal HA method for current data at $200 \mathrm{~m}$. Another fact found in the figure is that the harmonic results are less credible at both ends compared to the middle parts because of the interpolation of cubic splines. In another words, the harmonic results are more reliable during the middle parts of the sample time.

In Fig. 11 the current fit obtained from the three HA algorithms are compared to the observed power spectra. On account of nonlinear distortions of the four constituents, overtides such as $\mathrm{MO}_{3}$ can be discovered in the observed spectrum near the third and fourth diurnal bands, but their spectra are much smaller than those in diurnal and semidiurnal bands (note the log scale of the $y$ axis in Fig. 11a). The overview of the spectra (Fig. 11a) demonstrates that all three algorithms reveal the spectrum peaks at the bands around the selected six principal constituents. The performances of EHA and splined SHA are better than that of CHA, which indicates the necessity to apply HA with time-varying harmonic parameters. When we zoom in to the lowfrequency band (Fig. 11b), the comparison between the results of the three algorithms tells us that the EHA fit encompasses more of the observed signal energy than the other two algorithms at low frequencies. In the diurnal and semidiurnal bands (Figs. 11c,d), the EHA fit almost perfectly coincides with the observed spectrum and reproduces the cusp-like shapes formed around the dominant frequencies- $\mathrm{M}_{2}, \mathrm{~S}_{2}, \mathrm{~K}_{1}$, and $\mathrm{O}_{1}$-with great accuracy, compared with results of the splined SHA and CHA. The results of the power spectra indicate that $\mathrm{M}_{2}, \mathrm{~S}_{2}, \mathrm{~K}_{1}$, and $\mathrm{O}_{1}$ are definitely the most significant tidal constituents in this region during the observing time. It agrees well with other studies in the same region. Moreover, this is additional evidence that shows the capability of EHA to obtain harmonic parameters and reveals the real observation data.

The overall temporal variations of amplitudes for all constituents are significant during the selected time period, which indicates obvious seasonal variations for the four constituents. Based on the overall magnitude of amplitudes, the dominant constituents are $\mathrm{M}_{2}$ and $\mathrm{K}_{1}$ at 200-m depth at this observation site, which is consistent with studies around this area by other studies (Duda and Rainville 2008; Klymak et al. 2011; Lee et al. 2012; Ma et al. 2013; Vlasenko et al. 2010; Xu et al. 2013).
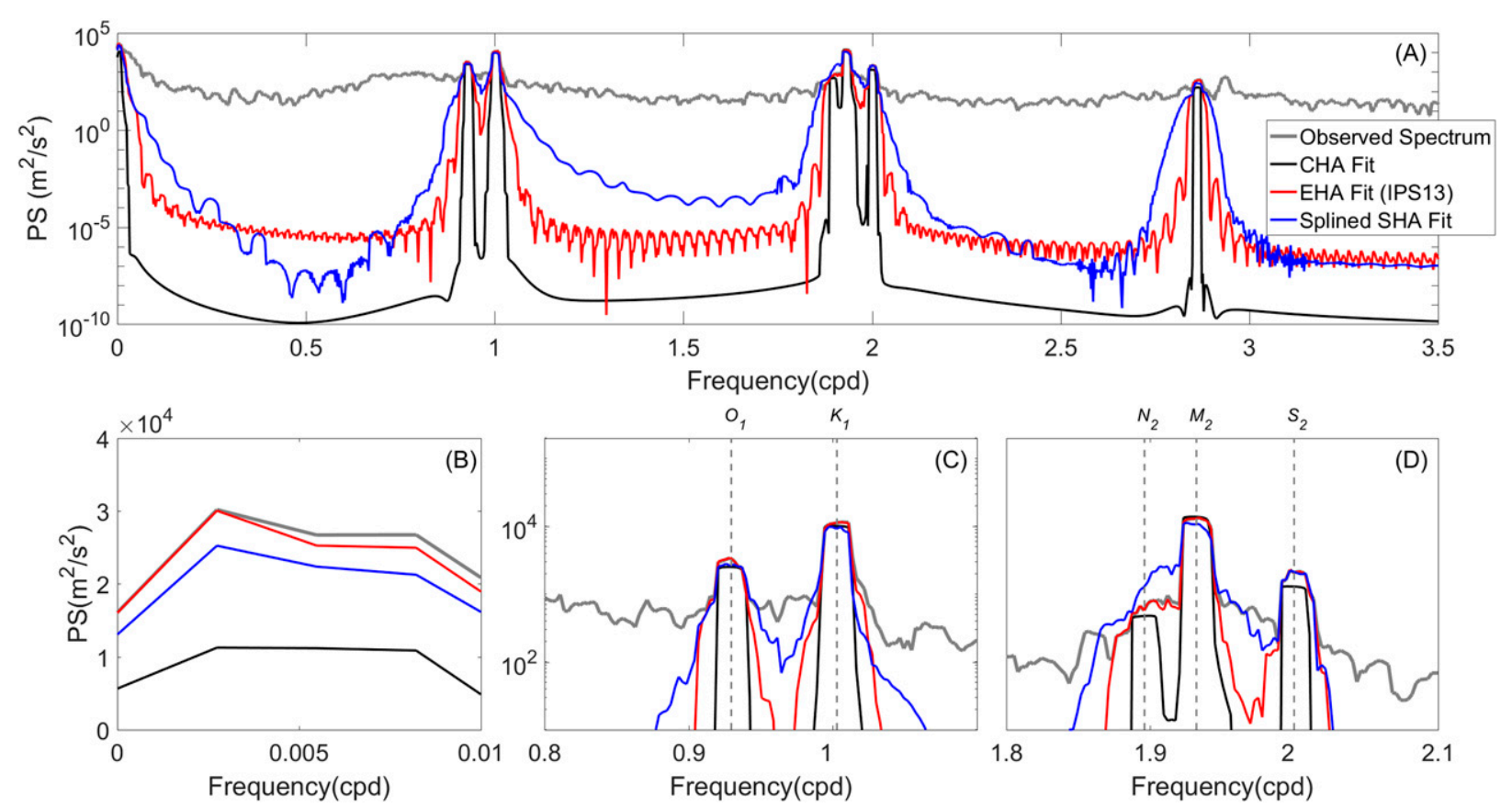

FIG. 11. (a) Power spectra of the observed signal (gray line) and current fit for all methods: CHA (black), EHA (IPS13) (red), and Splined SHA (blue). Zoomed-in views of the power spectra for the (b) low-frequency, (c) diurnal and (d) semidiurnal bands. 
Moreover, the temporal variabilities for these four principal tidal constituents are apparent, so the harmonic constants obtained by CHA cannot reveal the details of variabilities for internal tidal currents.

\section{Summary}

This paper presented an enhanced harmonic analysis algorithm that obtains harmonic parameters with continuous temporal variations. The whole process of methodology for this algorithm is derived based on the IPS and cubic spline interpolation method. Using numerical examples, the accuracy of EHA is verified. It has the ability to reveal the continuous and smooth temporal variations of harmonic parameters for different tidal constituents of the prescribed current data. The mean difference between EHA and SHA lies in the fact that the former is using the data from the entire record to determine the harmonic parameters at any particular point with the spline coupling included. Then it is applied to 14 months of current data from a mooring located at the continental shelf of the northeastern SCS. Different IPSs lead to different harmonic results. We determine the optimal IPS for a time series by evaluating the misfits between the tidal predictions and the simulated data, as well as the consistency among different IPSs. Similar with CHA, EHA is carried out without the consideration of the dynamics of internal tides, assuming only the candidate tidal constituents. The advantage is to allow post facto analysis on the dynamics without any dynamic assumptions about the harmonic analysis. As a result, this approach may not be entirely adequate for strongly nonstationary or dynamically complex issues like internal solitary waves. In summary, EHA is a new viewpoint and a more precise method than CHA to view the temporal variations of harmonic parameters for internal tides (currents). While this method is applied here to a scalar time series of currents, it could be applied to any scalar time series, such as elevation, temperature, or salinity. Moreover, whether it can be extended to a twodimensional vector time series [e.g., $(u, v)]$ with surface splines or the two components should be treated separately with regular spline method requires a detailed dynamic analysis. When processes are linear, the combined harmonic analysis on two components make sense. But this does not necessarily apply in the nonlinear world. Future works should be done to solve the automatic selection of the IPS and to apply this method to observing data at different locations of the world's oceans.

Acknowledgments and data. The authors express their sincere gratitude to the South China Sea Mooring Array and the Ocean and Atmosphere Data Center for providing the mooring observations. We gratefully thank three anonymous reviewers for their insightful comments, which helped to promote the work and our understanding in this area. The data and the codes used in this paper are all available by contacting the corresponding author Prof. Lv. Partial support for this research was provided by the National Science and Technology Major Project through Grant 2016ZX05057015, the Ocean Engineering and Equipment ProjectIndependent Research and Development on Oilfield Production Equipment TLP under 500 meters-Research on Internal Wave Forecast Methods and Monitor Systems through Grant CCL2015SKGF0008, the Three Big Constructions: Supercomputing Application Cultivation Projects, the National Natural Science Foundation of China through Grants 41606006 and 41371496, the National Key Research and Development Plan through Grant 2016YFC1402304, the National Key Basic Research Program of China (Program 973) through Grant 2014CB745003, and the National Key Research and Development Program of China through Grant 2016YFC1402605.

Additionally, the authors declare no financial conflicts of interests. For more detailed information, please contact the corresponding author. Please note that there exists a different spelling for the first author Guangzhen Jin, which is Guang-Zhen Jin. And there exist different spellings for the corresponding author Xianqing Lv, which are Xian-Qing Lv, Xianqing Lu, and Xianqing Lü.

\section{APPENDIX}

\section{Derivations and Time Variabilities}

\section{a. Derivation of cubic spline interpolation on harmonic parameters}

In this part the interpolation formulas $\mathbf{F}\left(t_{i}\right)$ from $\left[A\left(\tilde{t}_{k}\right), \phi\left(\tilde{t}_{k}\right)\right]$ to $\left[A\left(t_{i}\right), \phi\left(t_{i}\right)\right]$ are derived with the cubic spline interpolation method. In the following derivations, $A\left(t_{i}\right)$ and $\phi\left(t_{i}\right)$ will be denoted as $H_{i}=\left\{f\left(t_{i}\right) ; i=0\right.$, $1, \ldots, N\}$, and $A\left(\tilde{t}_{k}\right) \phi\left(\tilde{t}_{k}\right)$ will be denoted as $\tilde{H}_{k}=$ $\left\{f\left(\tilde{t}_{k}\right) ; k=0,1, \ldots, n\right\}$.We start by dividing a closed time interval $[a, b]$, which is the range of $t$, into $n$ parts uniformly as follows:

$$
a=\tilde{t}_{0}<\tilde{t}_{1}<\cdots<\tilde{t}_{n-1}<\tilde{t}_{n}=b .
$$

The approach to mathematically model the shape of such elastic rulers fixed by $n+1 \mathrm{kt}\left(1 \mathrm{kt}=0.51 \mathrm{~m} \mathrm{~s}^{-1}\right)$ is to interpolate between each pair of knots $\left(\tilde{t}_{k-1}, \tilde{H}_{k-1}\right)$ and $\left(\tilde{t}_{k}, \tilde{H}_{k}\right)$ with cubic polynomials. In another words, the main task of this subsection is to derive the formulation of $\left\{H_{i} ; i=0,1, \ldots, N\right\}$ with values on IPs, that is, $\left\{\tilde{H}_{k} ; k=0,1, \ldots, n\right\}$. 
At times $\tilde{t}_{k}$ and $\tilde{t}_{k+1}$, for each subtime interval $\left[\tilde{t}_{k}, \tilde{t}_{k+1}\right]$, we denote the one-stage derivative of $f(t)$ as
Based on the spline interpolation method, for each $t \in\left[\tilde{t}_{k}, \tilde{t}_{k+1}\right], f(t)$ can be written as $f^{\prime}\left(\tilde{t}_{k}\right)=m_{k}, \quad f^{\prime}\left(\tilde{t}_{k+1}\right)=m_{k+1}, \quad k=0,1, \ldots, n-1$.

$$
\begin{aligned}
f(t)= & \left(1+2 \frac{t-\tilde{t}_{k}}{\tilde{t}_{k+1}-\tilde{t}_{k}}\right)\left(\frac{t-\tilde{t}_{k}}{\tilde{t}_{k+1}-\tilde{t}_{k}}\right)^{2} \tilde{H}_{k}+\left(1+2 \frac{t-\tilde{t}_{k+1}}{\tilde{t}_{k}-\tilde{t}_{k+1}}\right)\left(\frac{t-\tilde{t}_{k}}{\tilde{t}_{k+1}-\tilde{t}_{k}}\right)^{2} \tilde{H}_{k+1} \\
& +\left(t-\tilde{t}_{k}\right)\left(\frac{t-\tilde{t}_{k+1}}{\tilde{t}_{k}-\tilde{t}_{k+1}}\right)^{2} m_{k}+\left(t-\tilde{t}_{k+1}\right)\left(\frac{t-\tilde{t}_{k}}{\tilde{t}_{k+1}-\tilde{t}_{k}}\right)^{2} m_{k+1} .
\end{aligned}
$$

Next, we will try to express $m_{k}$ as a function of $\tilde{H}_{k}$ so that $f(t)$ can be written as a function of $\tilde{H}_{k}$ for each $t \in[a, b]$. Based on Eq. (A3), let $d t=\tilde{t}_{k+1}-\tilde{t}_{k}$ and take a twostage derivative of $f(t)$ for $t$. Then, we get

$$
\begin{aligned}
f^{\prime \prime}(t)= & {\left[\frac{6}{d t^{2}}-\frac{12}{d t^{3}}\left(\tilde{t}_{k+1}-t\right)\right] \tilde{H}_{k}+\left[\frac{6}{d t^{2}}-\frac{12}{d t^{3}}\left(t-\tilde{t}_{k}\right)\right] \tilde{H}_{k+1} } \\
& +\left[\frac{2}{d t}-\frac{6}{d t^{2}}\left(\tilde{t}_{k+1}-t\right)\right] m_{k}-\left[\frac{2}{d t}-\frac{6}{d t^{2}}\left(t-\tilde{t}_{k}\right)\right] m_{k+1} .
\end{aligned}
$$

In fact, the two-stage derivative of the cubic piecewise function $f^{\prime \prime}(t)$ should be continuous, which means the two-stage progressive derivative, that is,

$f^{\prime \prime}\left(\tilde{t}_{k}^{+}\right)=-\frac{6}{d t^{2}} \tilde{H}_{k}+\frac{6}{d t^{2}} \tilde{H}_{k+1}-\frac{4}{d t} m_{k}-\frac{2}{d t} m_{k+1}$,

and the left-hand derivative, that is,

$f^{\prime \prime}\left(\tilde{t}_{k}^{-}\right)=\frac{6}{d t^{2}} \tilde{H}_{k-1}+\frac{6}{d t^{2}} \tilde{H}_{k}+\frac{2}{d t} m_{k-1}+\frac{4}{d t} m_{k}$,

are equal: $f^{\prime \prime}\left(\tilde{t}_{k}^{+}\right)=f^{\prime \prime}\left(\tilde{t}_{k}^{-}\right)$.

Then, we get the system of linear equations for the one-stage derivative $m$ :

$$
\begin{aligned}
& \left(1-\alpha_{k}\right) m_{k-1}+2 m_{k}+\alpha_{k} m_{k+1}=\beta_{k}, \quad \text { for } \\
& \quad k=1,2, \ldots, n-1,
\end{aligned}
$$

in which $\alpha_{k}=1 / 2$ and $\beta_{k}=(3 / 2 d t)\left(\tilde{H}_{k+1}-\tilde{H}_{k-1}\right)$.

Add natural boundary conditions, $f^{\prime \prime}\left(\tilde{t}_{0}\right)=f^{\prime \prime}\left(\tilde{t}_{n}\right)=0$, which can be written as

$$
\left\{\begin{array}{c}
2 m_{0}+m_{1}=\frac{3}{d t}\left(\tilde{H}_{1}-\tilde{H}_{0}\right) \\
m_{n-1}+2 m_{n}=\frac{3}{d t}\left(\tilde{H}_{n}-\tilde{H}_{n-1}\right)
\end{array}\right.
$$

in Eqs. (A7). Then, we get a system of linear equations that has a unique solution. For convenience, the system can be reduced to matrix form, that is,

$$
\mathbf{A}_{\alpha} \mathbf{M}=\mathbf{b}_{\beta} \tilde{\mathbf{H}}
$$

where

$\mathbf{A}_{\alpha}=\left[\begin{array}{cccccccc}2 & 1 & 0 & 0 & \cdots & 0 & 0 & 0 \\ 0.5 & 2 & 0.5 & 0 & \cdots & 0 & 0 & 0 \\ 0 & 0.5 & 2 & 0.5 & \cdots & 0 & 0 & 0 \\ \vdots & \vdots & \vdots & \vdots & \cdots & \vdots & \vdots & \vdots \\ 0 & 0 & 0 & 0 & \cdots & 0.5 & 2 & 0.5 \\ 0 & 0 & 0 & 0 & \cdots & 0 & 1 & 2\end{array}\right]_{(n+1) \times(n+1)}$

$\mathbf{M}=\left(m_{0}, m_{1}, \ldots, m_{n}\right)^{\mathrm{T}}, \quad \tilde{\mathbf{H}}=\left(\tilde{H}_{0}, \tilde{H}_{1}, \ldots, \tilde{H}_{n}\right)^{\mathrm{T}}, \quad$ and

$\mathbf{b}_{\beta}=\frac{3}{2 d t}\left[\begin{array}{cccccccc}-2 & 2 & 0 & 0 & \cdots & 0 & 0 & 0 \\ -1 & 0 & 1 & 0 & \cdots & 0 & 0 & 0 \\ 0 & -1 & 0 & 1 & \cdots & 0 & 0 & 0 \\ \vdots & \vdots & \vdots & \vdots & \cdots & \vdots & \vdots & \vdots \\ 0 & 0 & 0 & 0 & \cdots & -1 & 0 & 1 \\ 0 & 0 & 0 & 0 & \cdots & 0 & -2 & 2\end{array}\right]_{(n+1) \times(n+1)}$

Then, Eq. (A9) can be easily solved and the matrix for the one-stage derivative $\mathbf{M}$ can be finally expressed as a function of $\tilde{\mathbf{H}}$ :

$$
\mathbf{M}=\left(\mathbf{A}_{\alpha}^{-1} \mathbf{b}_{\beta}\right) \tilde{\mathbf{H}}
$$

Combining Eqs. (A3) and (A12), we obtain the timevarying tidal (current) constant $f(t)$ for any $t \in[a, b]$ as a linear combination of constants at IPs, that is, $\left\{\tilde{H}_{k} ; k=\right.$ $0,1, \ldots, n\}$. The function of $f(t)$ will be represented in the following form, which is convenient for formula derivations in the next section. For a specific $t \in\left[\tilde{t}_{k}, \tilde{t}_{k+1}\right]$, 

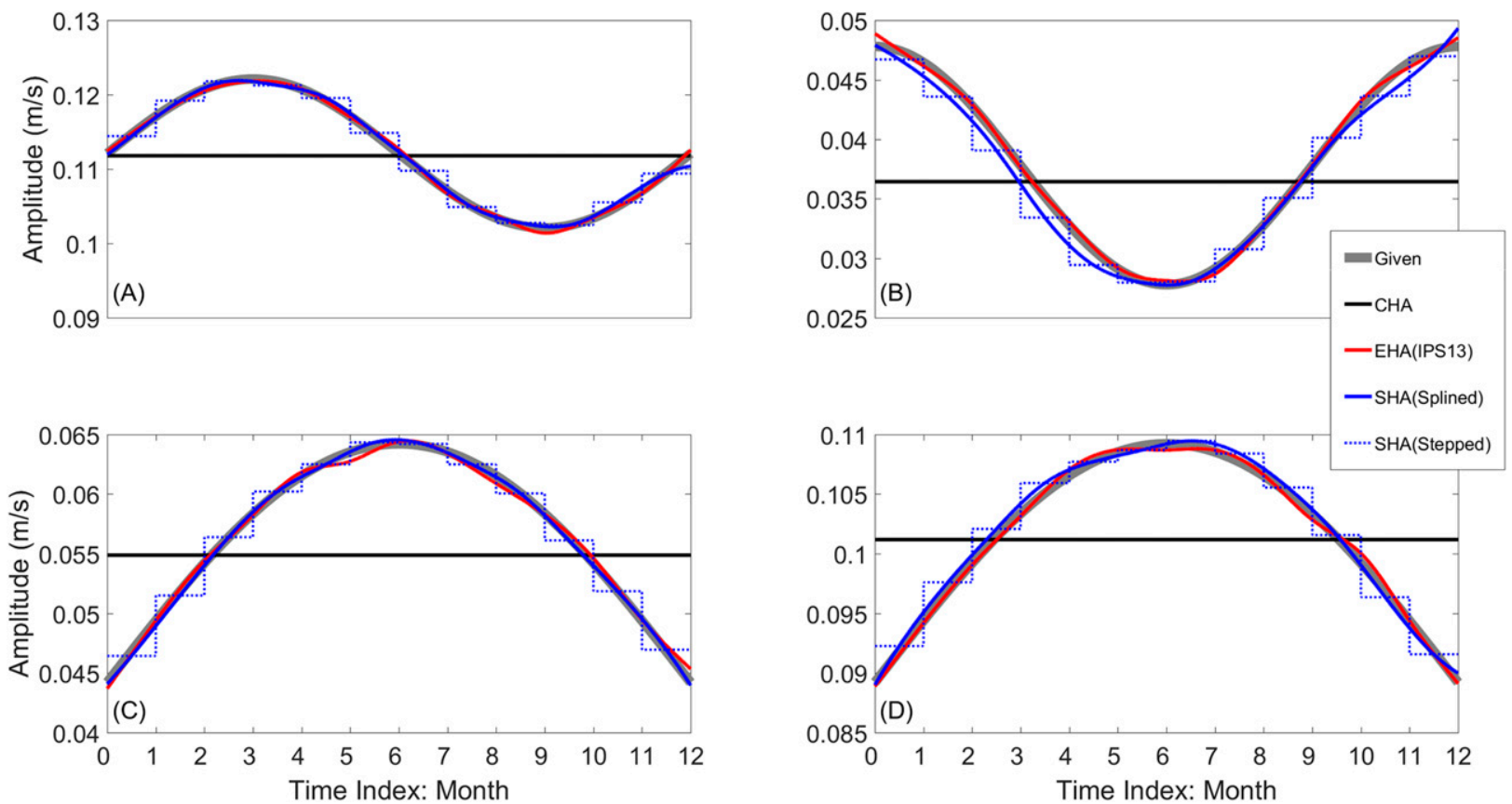

FIG. A1. Given values and HA results of the current amplitudes for the four selected tidal constituents: (a) $\mathrm{M}_{2}$, (b) $\mathrm{S}_{2}$, (c) $\mathrm{O}_{1}$, and (d) $\mathrm{K}_{1}$. The horizontal gray lines are the given values; the black line is the CHA, the red is the EHA, the solid blue is the SHA (Splined), and the dotted-stepped blue is the SHA (Stepped).

$$
\begin{aligned}
f(t)= & A(t) \tilde{H}_{k}+B(t) \tilde{H}_{k+1}+C(t)\left(\mathbf{A}_{\alpha}^{-1} \mathbf{b}_{\beta}\right)_{k+1} \\
& \times \tilde{H}_{k}+D(t)\left(\mathbf{A}_{\alpha}^{-1} \mathbf{b}_{\beta}\right)_{k+1} \cdot \tilde{H}_{k+1} \\
\triangleq & \mathbf{F}_{k+1} \cdot \tilde{H}\left[\tilde{t}_{k}, \tilde{t}_{k+1}\right], \\
A(t)= & \left(1+2 \frac{t-\tilde{t}_{k}}{\tilde{t}_{k+1}-\tilde{t}_{k}}\right)\left(\frac{t-\tilde{t}_{k}}{\tilde{t}_{k+1}-\tilde{t}_{k}}\right)^{2}, \\
B(t)= & \left(1+2 \frac{t-\tilde{t}_{k+1}}{\tilde{t}_{k}-\tilde{t}_{k+1}}\right)\left(\frac{t-\tilde{t}_{k}}{\tilde{t}_{k+1}-\tilde{t}_{k}}\right)^{2}, \text { where } \\
C(t)= & \left(t-\tilde{t}_{k}\right)\left(\frac{t-\tilde{t}_{k+1}}{\tilde{t}_{k}-\tilde{t}_{k+1}}\right)^{2}, \text { and } \\
D(t)= & \left(t-\tilde{t}_{k+1}\right)\left(\frac{t-\tilde{t}_{k}}{\tilde{t}_{k+1}-\tilde{t}_{k}}\right)^{2} ;
\end{aligned}
$$

the subscript marked $k+1$ indicates the $(k+1)$ th row of the corresponding matrix; and $\mathbf{F}$ is the transformation matrix with $n$ rows and $n+1$ columns.

\section{b. Time variabilities of phases, mean flows, and confidence intervals}

Accompanying Fig. 2, we provided the harmonic results of all four selected constituents in section 3 in Figs. A1 and A2. Similar to the examples of $\mathrm{M}_{2}$ and $\mathrm{K}_{1}$ in the text, all the algorithms except for CHA have the ability to reveal time-varying signals of harmonic parameters from the prescribed series. EHA with IPS13 generally performs better than the other algorithms.

Accompanying Fig. 7, the phases for different constituents are obtained by the three algorithms. Figure A3 illustrates the current phases as functions of time for the four principal constituents, which are $\mathrm{M}_{2}, \mathrm{~K}_{1}, \mathrm{~S}_{2}$, and $\mathrm{O}_{1}$. The values of the constant phases are $129.5^{\circ}, 114.8^{\circ}$, $183.3^{\circ}$, and $294.8^{\circ}$ for the corresponding constituents, respectively. To compare between different results, all the phases are adjusted by adding or subtracting $360^{\circ}$. It is a noteworthy phenomenon that the differences between time-varying phases and the referenced constant phases are different for the four constituents. In general, the EHA results show that $\mathrm{M}_{2}$ and $\mathrm{K}_{1}$ exhibit small ranges of variations. The differences between them and the CHA results are also small. However, the ranges and differences are both larger for the $\mathrm{S}_{2}$ and $\mathrm{O}_{1}$ constituents. During most of observing time, $\mathrm{M}_{2}$ and $\mathrm{S}_{2}$ are in phase, indicating their current amplitude being added to each other. On the contrary, $\mathrm{K}_{1}$ and $\mathrm{O}_{1}$ are out of phase, indicating their current amplitude being decreased to each other. Although this issue is interesting and noteworthy, it is beyond the scope of present study. For now we only provide the estimates of phases obtained by the three HA algorithms in this paper. More analyses will be carried out in the near future. 

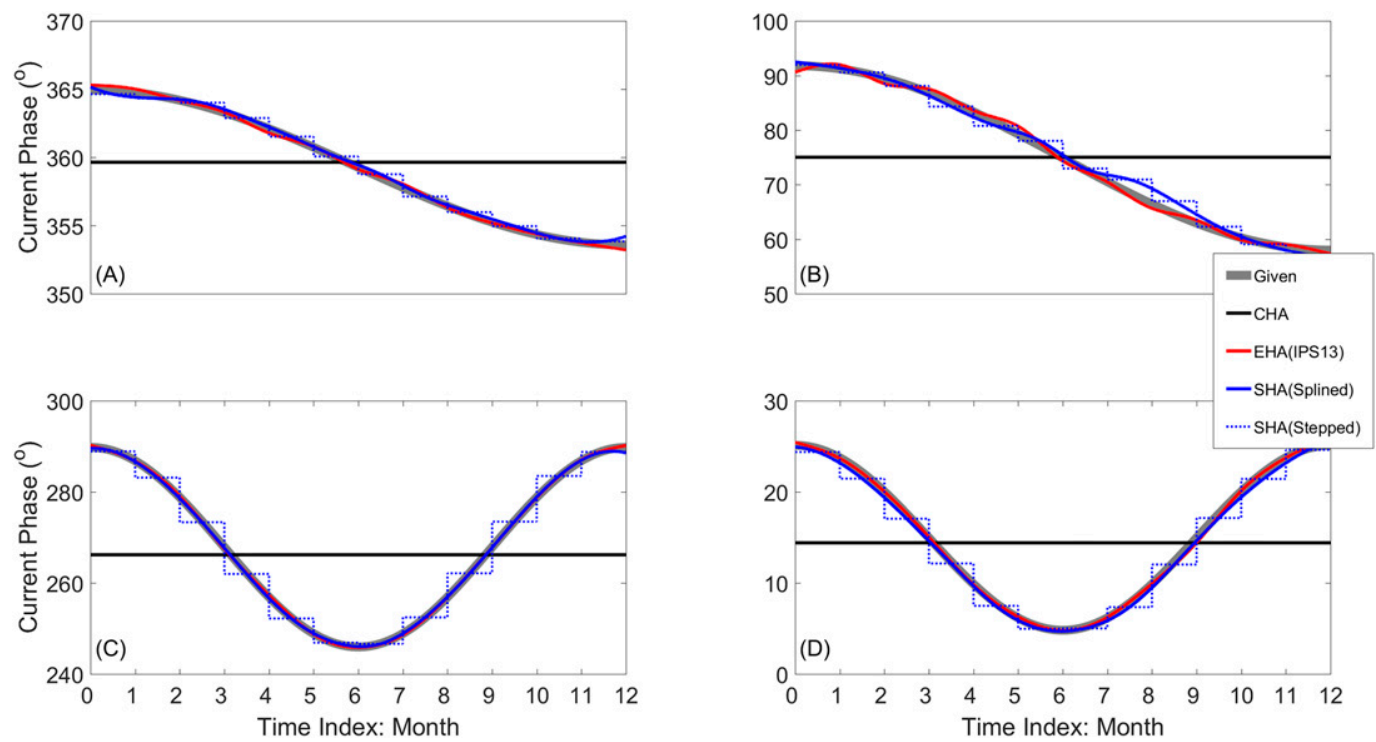

FIG. A2. As in Fig. A1, but for the current phases.

Figure A4 presents the time-varying mean flow obtained by different HA algorithms. The result of $\mathrm{CHA}$ provides us the overall mean flow, which is $-0.0508 \mathrm{~m} \mathrm{~s}^{-1}$. However, according to the temporal variations in the figure, the mean flow at the depth of $200 \mathrm{~m}$ at this observation site has obvious annual variations. Despite the overfitting for cubic spline interpolation, the variations of the mean flow will be
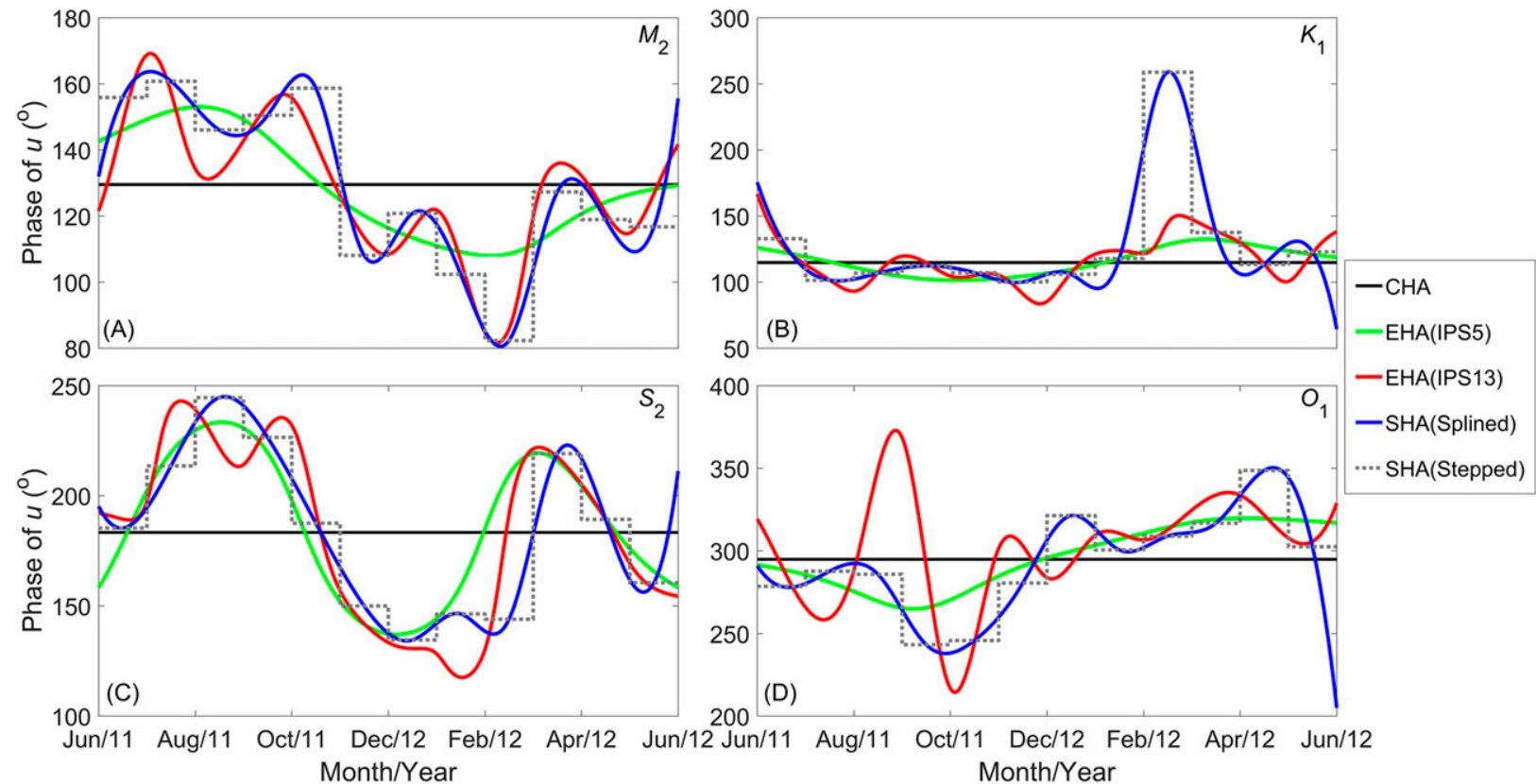

FIG. A3. Results of the time-varying zonal current phases obtained by three HA algorithms at 200-m depth. The principal tidal constituents (a) $\mathrm{M}_{2}$, (b) $\mathrm{K}_{1}$, (c) $\mathrm{S}_{2}$, and (d) $\mathrm{O}_{1}$. The horizontal black lines are the CHA, the green is the EHA (IPS5), the red is the 1EHA (IPS13), the blue is the SHA (Splined), and the dashed-stepped gray is the SHA (Stepped). 


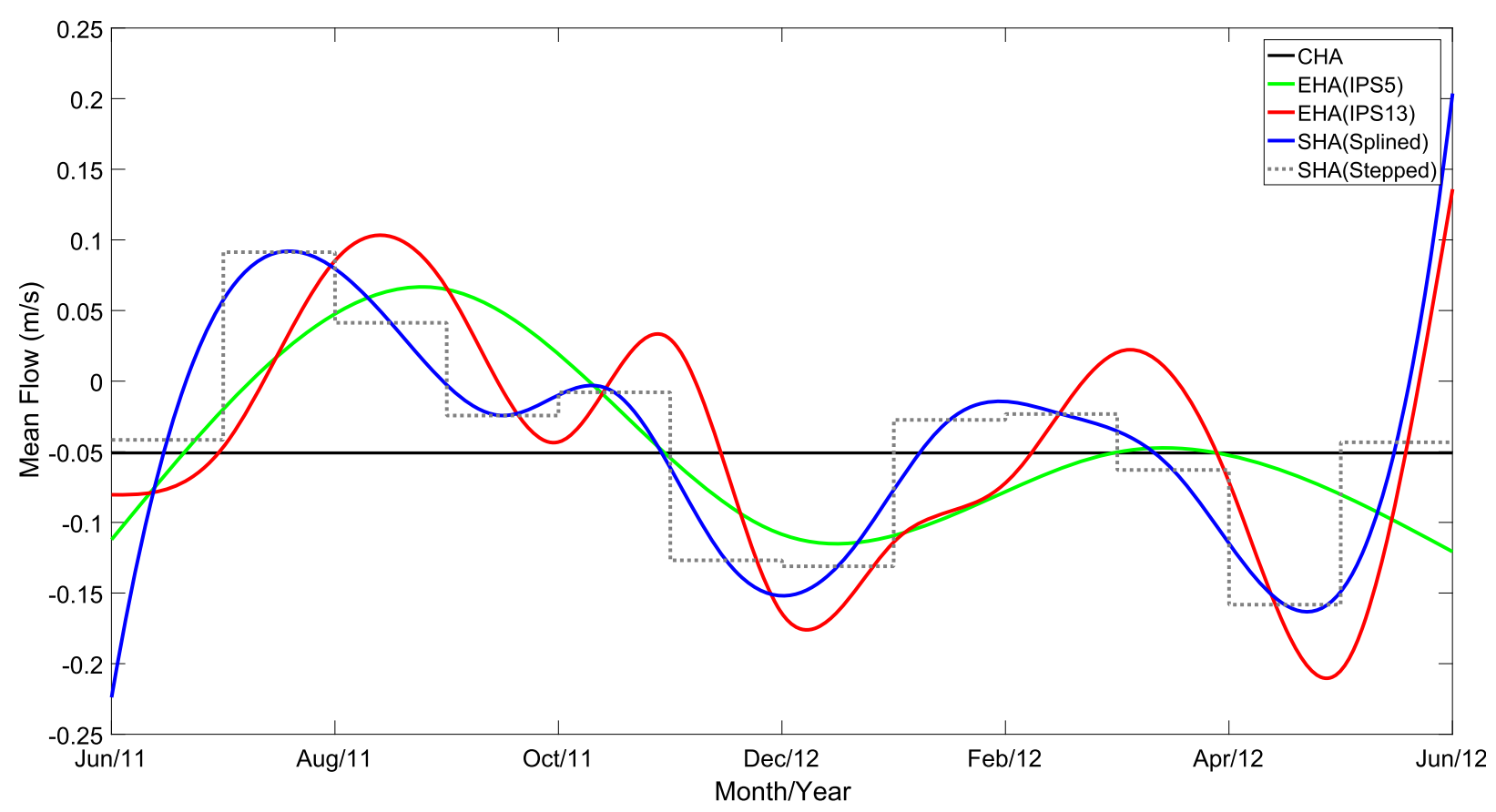

FIG. A4. As in Fig. A3, but for the mean flows.

approximately $0.1800 \mathrm{~m} \mathrm{~s}^{-1}$, which is quite large compared with the overall mean flow. The general variations obtained by SHA and EHA are consistent with each other. The overall mean flow is stronger in summer than in winter, which has been investigated by Xu et al. (2013).
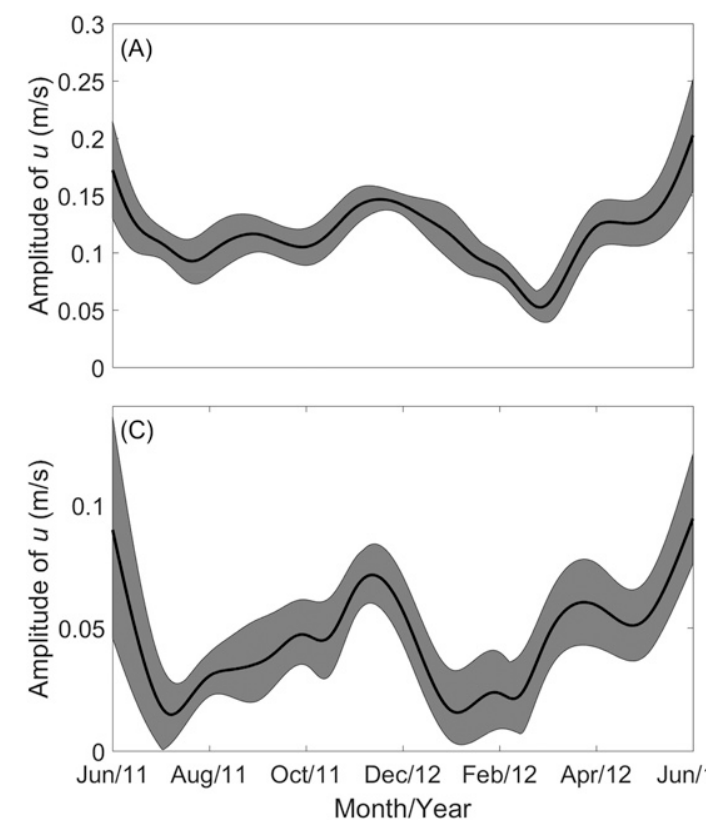

FIG. A5. Results of the 95\% CI (gray band) for time-varying zonal current amplitudes obtained by EHA (IPS13) at 200-m depth. The principal tidal constituents (a) $\mathrm{M}_{2}$, (b) $\mathrm{K}_{1}$, (c) $\mathrm{S}_{2}$, and (d) $\mathrm{O}_{1}$.
With a bootstrap technique, the time-varying $95 \%$ confidence intervals for the harmonic parameters are calculated and illustrated in Fig. A5. Only results obtained by EHA (IPS13) are provided as an example in the figure. Note that the confidence intervals at both ends of the harmonic parameters are larger than those in
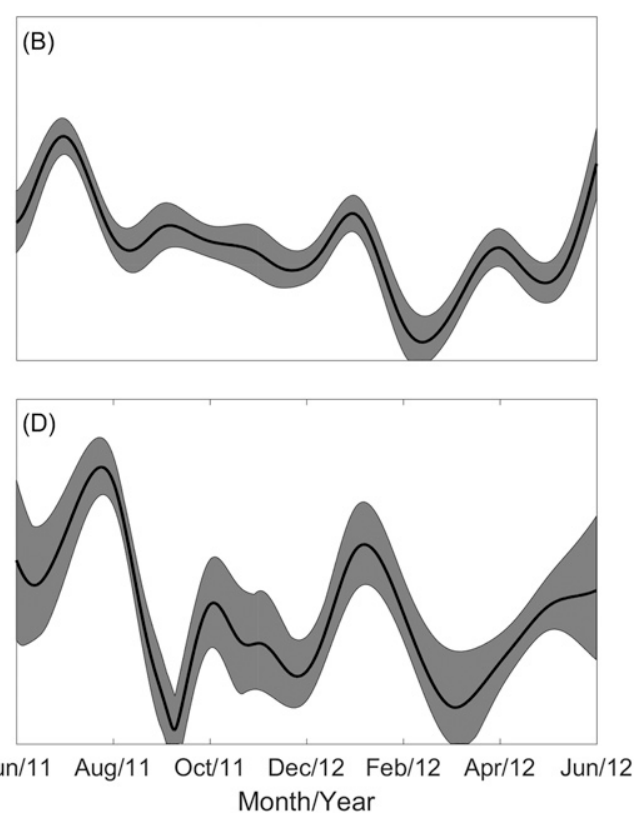
the middle of the time series, which means less confidence than the results in the middle parts. Thus, an important note for users is that the harmonic results at both ends of the sample time should be used with great care.

\section{REFERENCES}

Alford, M. H., and Coauthors, 2015: The formation and fate of internal waves in the South China Sea. Nature, 521, 65-69, https://doi.org/10.1038/nature14399.

Amin, M., 1983: On perturbations of harmonic constants in the Thames Estuary. Geophys. J. Int., 73, 587-603, https://doi.org/ 10.1111/j.1365-246X.1983.tb03334.x.

- 1985: Temporal variations of tides on the west coast of Great Britain. Geophys. J. Int., 82, 279-299, https://doi.org/10.1111/ j.1365-246X.1985.tb05138.x.

- 1993: The mutual influence of tidal constituents in the presence of bottom stress. Estuarine Coastal Shelf Sci., 37, 625-633, https://doi.org/10.1006/ecss.1993.1078.

Baines, P., 1973: The generation of internal tides by flat-bump topography. Deep-Sea Res. Oceanogr. Abstr., 20, 179-205, https://doi.org/10.1016/0011-7471(73)90050-8.

Buijsman, M., J. McWilliams, and C. Jackson, 2010: East-west asymmetry in nonlinear internal waves from Luzon Strait. J. Geophys. Res., 115, C10057, https://doi.org/10.1029/ 2009JC006004.

Buschman, F., A. Hoitink, M. van der Vegt, and P. Hoekstra, 2009: Subtidal water level variation controlled by river flow and tides. Water Resour. Res., 45, W10420, https://doi.org/10.1029/ 2009WR008167.

Chavanne, C., P. Flament, G. Carter, M. Merrifield, D. Luther, E. Zaron, and K. Gurgel, 2010: The surface expression of semidiurnal internal tides near a strong source at Hawaii. Part I: Observations and numerical predictions. J. Phys. Oceanogr., 40, 1155-1179, https://doi.org/10.1175/ 2010JPO4222.1.

Codiga, D. L., 2011: Unified tidal analysis and prediction using the UTide Matlab functions. Graduate School of Oceanography, University of Rhode Island Rep. 2011-01, 59 pp.

Colosi, J. A., and W. Munk, 2006: Tales of the venerable Honolulu tide gauge. J. Phys. Oceanogr., 36, 967-996, https://doi.org/ 10.1175/JPO2876.1.

Darwin, G. H., 1891: Bakerian lecture-On tidal prediction. Proc. Roy. Soc. London, 49, 130-133, https://doi.org/10.1098/ rspl.1890.0075.

de Boor, C., 1978: A Practical Guide to Splines. Applied Mathematical Sciences, Vol. 27, Springer-Verlag, 348 pp.

Desquilbet, L., and F. Mariotti, 2010: Dose-response analyses using restricted cubic spline functions in public health research. Stat. Med., 29, 1037-1057, https://doi.org/10.1002/sim.3841.

DiCiccio, T., and B. Efron, 1996: Bootstrap confidence intervals (with discussion). Stat. Sci., 11, 189-228, https://doi.org/ $10.1214 / \mathrm{ss} / 1032280214$

DiLorenzo, J. L., P. Huang, M. L. Thatcher, and T. O. Najarian, 1993: Dredging impacts on Delaware Estuary tides. Estuarine and Coastal Modeling, M. Spaulding et al., Eds., American Society of Civil Engineers, 86-104.

Duda, T. F., and L. Rainville, 2008: Diurnal and semidiurnal internal tide energy flux at a continental slope in the South China Sea. J. Geophys. Res., 113, C03025, https://doi.org/ 10.1029/2007JC004418.
Efron, B., and R. J. Tibshirani, 1994: An Introduction to the Bootstrap. Monographs on Statistics and Applied Probability, Vol. 57, CRC Press, 456 pp.

Foreman, M. G. G., 1977: Manual for tidal heights analysis and prediction. Institute of Ocean Sciences Pacific Marine Science Rep. 77-10, 58 pp.

— and R. Henry, 1989: The harmonic analysis of tidal model time series. Adv. Water Resour., 12, 109-120, https://doi.org/ 10.1016/0309-1708(89)90017-1.

_ J. Y. Cherniawsky, and V. A. Ballantyne, 2009: Versatile harmonic tidal analysis: Improvements and applications. J. Atmos. Oceanic Technol., 26, 806-817, https://doi.org/ 10.1175/2008JTECHO615.1.

Gao, D., G. Jin, and X. Lü, 2016: Temporal variations in internal tide multimodal structure on the continental shelf, South China Sea. Chin. J. Oceanol. Limnol., 35, 70-78, https:// doi.org/10.1007/s00343-016-5168-0.

Gao, X., Z. Wei, X. Lv, Y. Wang, and G. Fang, 2015: Numerical study of tidal dynamics in the South China Sea with adjoint method. Ocean Modell., 92, 101-114, https://doi.org/10.1016/ j.ocemod.2015.05.010.

Gao, Y., G. Jin, H. Chen, and X. Lv, 2013: Estimation of open boundary conditions based on an isopycnic-coordinate internal tidal model with adjoint assimilation method. Math Probl. Eng., 2013, 461-461, https://doi.org/10.1155/2013/ 321387.

Gerkema, T., F. P. A. Lam, and L. R. Maas, 2004: Internal tides in the Bay of Biscay: Conversion rates and seasonal effects. Deep-Sea Res. II, 51, 2995-3008, https://doi.org/10.1016/ j.dsr2.2004.09.012.

Godin, G., 1972: The Analysis of Tides. University of Toronto Press, $264 \mathrm{pp}$.

- 1983: On the predictability of currents. Int. Hydrogr. Rev., 60, 119-126.

- 1985: Modification of river tides by the discharge. J. Waterw. Port Coastal Ocean Eng., 111, 257-274, https://doi.org/ 10.1061/(ASCE)0733-950X(1985)111:2(257).

, 1999: The propagation of tides up rivers with special considerations on the upper Saint Lawrence River. Estuarine Coastal Shelf Sci., 48, 307-324, https://doi.org/10.1006/ ecss.1998.0422.

Groves, G. W., and B. D. Zetler, 1964: The cross spectrum of sea level at San Francisco and Honolulu. J. Mar. Res., 22, 269-275.

Guan, S., W. Zhao, J. Huthnance, J. Tian, and J. Wang, 2014: Observed upper ocean response to typhoon Megi (2010) in the Northern South China Sea. J. Geophys. Res. Oceans, 119, 3134-3157, https://doi.org/10.1002/2013JC009661.

Guo, P., W. Fang, Z. Gan, R. Chen, and X. Long, 2006: Internal tide characteristics over northern South China Sea continental slope. Chin. Sci. Bull., 51, 17-25, https://doi.org/10.1007/ s11434-006-9017-y.

,-- C. Liu, and F. Qiu, 2012: Seasonal characteristics of internal tides on the continental shelf in the northern South China Sea. J. Geophys. Res., 117, C04023, https://doi.org/ 10.1029/2011JC007215.

Guo, Z., H. Pan, W. Fan, and X. Lv, 2017: Application of surface spline interpolation in inversion of bottom friction coefficients. J. Atmos. Oceanic Technol., 34, 2021-2028, https:// doi.org/10.1175/JTECH-D-17-0012.1.

Heisenberg, W., 1927: Über den anschaulichen Inhalt der quantentheoretischen Kinematik und Mechanik. Z. Phys., 43, 172-198, https://doi.org/10.1007/BF01397280. 
Helland-Hansen, B., and F. Nansen, 1909: The Norwegian Sea: Its physical oceanography based upon the Norwegian researches 1900-1904. Report on Norwegian Fishery and Marine Investigations, Vol. 2, No. 2, Kristiania Malling, 390 pp.

Horsburgh, K., and C. Wilson, 2007: Tide-surge interaction and its role in the distribution of surge residuals in the North Sea. J. Geophys. Res., 112, C08003, https://doi.org/10.1029/ 2006JC004033.

Hu, E. A., A. Pan, V. Malik, and Q. Sun, 2012: White rice consumption and risk of type 2 diabetes: Meta-analysis and systematic review. BMJ (Br. Med. J.), 344, e1454, https://doi.org/ 10.1136/bmj.e1454.

Jan, S., R.-C. Lien, and C.-H. Ting, 2008: Numerical study of baroclinic tides in Luzon Strait. J. Oceanogr., 64, 789-802, https:// doi.org/10.1007/s10872-008-0066-5.

Jay, D. A., 1991: Green's law revisited: Tidal long-wave propagation in channels with strong topography. J. Geophys. Res., 96, 20 585-20 598, https://doi.org/10.1029/91JC01633.

- 2009: Evolution of tidal amplitudes in the eastern Pacific Ocean. Geophys. Res. Lett., 36, L04603, https://doi.org/ 10.1029/2008GL036185.

— , and E. P. Flinchem, 1995: Wavelet transform analyses of nonstationary tidal currents. Proceedings of the IEEE Fifth Working Conference on Current Measurement, S. P. Anderson, G. F. Appell, and A. J. Williams III, Eds., IEEE, 100-105, https:// doi.org/10.1109/CCM.1995.516158.

— , and — 1997: Interaction of fluctuating river flow with a barotropic tide: A demonstration of wavelet tidal analysis methods. J. Geophys. Res., 102, 5705-5720, https://doi.org/ 10.1029/96JC00496.

—, and _ 1999: A comparison of methods for analysis of tidal records containing multi-scale non-tidal background energy. Cont. Shelf Res., 19, 1695-1732, https://doi.org/10.1016/S02784343(99)00036-9.

_- and T. Kukulka, 2003: Revising the paradigm of tidal analysis-The uses of non-stationary data. Ocean Dyn., 53, 110-125, https://doi.org/10.1007/s10236-003-0042-y.

__ K. K. Leffler, and S. Degens, 2011: Long-term evolution of Columbia River tides. J. Waterw. Port Coastal Ocean Eng., 137, 182-191, https://doi.org/10.1061/(ASCE)WW.19435460.0000082.

Jin, G., Q. Liu, and X. Lv, 2015: Inversion study of vertical eddy viscosity coefficient based on an internal tidal model with the adjoint method. Math. Probl. Eng., 2015, 915793, https:// doi.org/10.1155/2015/915793.

Kelly, S. M., N. L. Jones, G. N. Ivey, and R. J. Lowe, 2015: Internaltide spectroscopy and prediction in the Timor Sea. J. Phys. Oceanogr., 45, 64-83, https://doi.org/10.1175/JPO-D-14-0007.1.

Klymak, J. M., M. H. Alford, R. Pinkel, R.-C. Lien, Y. J. Yang, and T.-Y. Tang, 2011: The breaking and scattering of the internal tide on a continental slope. J. Phys. Oceanogr., 41, 926-945, https://doi.org/10.1175/2010JPO4500.1.

Kukulka, T., and D. A. Jay, 2003a: Impacts of Columbia River discharge on salmonid habitat: 1 . A nonstationary fluvial tide model. J. Geophys. Res., 108, 3293, https://doi.org/10.1029/ 2002JC001382.

—_, and —, 2003b: Impacts of Columbia River discharge on salmonid habitat: 2 . Changes in shallow-water habitat. J. Geophys. Res., 108, 3294, https://doi.org/10.1029/ 2003JC001829.

Lawn, J. E., and Coauthors, 2011: Stillbirths: Where? When? Why? How to make the data count? Lancet, 377, 1448-1463, https:// doi.org/10.1016/S0140-6736(10)62187-3.
Lee, I. H., Y.-H. Wang, Y. Yang, and D.-P. Wang, 2012: Temporal variability of internal tides in the northeast South China Sea. J. Geophys. Res., 117, C02013, https://doi.org/10.1029/ 2011JC007518.

Leffler, K. E., and D. A. Jay, 2009: Enhancing tidal harmonic analysis: Robust (hybrid $L^{1} / L^{2}$ ) solutions. Cont. Shelf Res., 29, 78-88, https://doi.org/10.1016/j.csr.2008.04.011.

Liu, J., S. Cai, and S. Wang, 2010: Currents and mixing in the northern South China Sea. Chin. J. Oceanol. Limnol., 28, 974-980, https://doi.org/10.1007/s00343-010-9094-2.

Lu, X., and J. Zhang, 2006: Numerical study on spatially varying bottom friction coefficient of a 2D tidal model with adjoint method. Cont. Shelf Res., 26, 1905-1923, https://doi.org/ 10.1016/j.csr.2006.06.007.

Ma, B. B., R.-C. Lien, and D. S. Ko, 2013: The variability of internal tides in the Northern South China Sea. J. Oceanogr., 69, 619-630, https://doi.org/10.1007/s10872-013-0198-0.

Malik, R., F. Zhou, and G. Ceder, 2011: Kinetics of nonequilibrium lithium incorporation in $\mathrm{LiFePO}_{4}$. Nat. Mater., 10, 587-590, https://doi.org/10.1038/nmat3065.

Matte, P., D. A. Jay, and E. D. Zaron, 2013: Adaptation of classical tidal harmonic analysis to nonstationary tides, with application to river tides. J. Atmos. Oceanic Technol., 30, 569-589, https://doi.org/10.1175/JTECH-D-12-00016.1.

_, Y. Secretan, and J. Morin, 2014: Temporal and spatial variability of tidal-fluvial dynamics in the St. Lawrence fluvial estuary: An application of nonstationary tidal harmonic analysis. J. Geophys. Res. Oceans, 119, 5724-5744, https://doi.org/10.1002/2014JC009791.

Munk, W. H., and E. Bullard, 1963: Patching the long-wave spectrum across the tides. J. Geophys. Res., 68, 3627-3634, https:// doi.org/10.1029/JZ068i012p03627.

_ on Oceanography: A Collection of Papers Dedicated to Koji Hidaka, University of Tokyo Press, 339-344.

Pan, H., Z. Guo, and X. Lv, 2017: Inversion of tidal open boundary conditions of the $\mathrm{M}_{2}$ constituent in the Bohai and Yellow Seas. J. Atmos. Oceanic Technol., 34, 1661-1672, https://doi.org/ 10.1175/JTECH-D-16-0238.1.

,-- Y Y. Wang, and X. Lv, 2018: Application of the EMD method to river tides. J. Atmos. Oceanic Technol., 35, 809-819, https://doi.org/10.1175/JTECH-D-17-0185.1.

Parker, B. B., 2007: Tidal analysis and prediction. NOAA Special Publ. NOS CO-OPS 3, 378 pp.

Pawlowicz, R., B. Beardsley, and S. Lentz, 2002: Classical tidal harmonic analysis including error estimates in MATLAB using T_TIDE. Comput. Geosci., 28, 929-937, https://doi.org/ 10.1016/S0098-3004(02)00013-4.

Plueddemann, A., and J. Farrar, 2006: Observations and models of the energy flux from the wind to mixed-layer inertial currents. Deep-Sea Res. II, 53, 5-30, https://doi.org/10.1016/j.dsr2.2005.10.017.

Qamar, I., 1993: Method to determine optimum number of knots for cubic splines. Int. J. Numer. Methods Biomed. Eng., 9, 483-488.

Ramp, S., Y. Yang, and F. Bahr, 2010: Characterizing the nonlinear internal wave climate in the northeastern South China Sea. Nonlinear Processes Geophys., 17, 481-498, https://doi.org/ 10.5194/npg-17-481-2010.

Reinsch, C. H., 1967: Smoothing by spline functions. Numer. Math., 10, 177-183, https://doi.org/10.1007/BF02162161.

Schoenberg, I. J., 1964: On interpolation by spline functions and its minimal properties. On Approximation Theory (in German), P. L. Butzer and J. Korevaar, Eds., Springer, 109-129.

Shaw, P.-T., D. S. Ko, and S.-Y. Chao, 2009: Internal solitary waves induced by flow over a ridge: With applications to the northern 
South China Sea. J. Geophys. Res., 114, C02019, https:// doi.org/10.1029/2008JC005007.

Teague, W. J., E. Jarosz, D. W. Wang, and D. A. Mitchell, 2007: Observed oceanic response over the upper continental slope and outer shelf during Hurricane Ivan. J. Phys. Oceanogr., 37, 2181-2206, https://doi.org/10.1175/JPO3115.1.

The 1000 Genomes Project Consortium, 2015: A global reference for human genetic variation. Nature, 526, 68-74, https://doi.org/ 10.1038/nature15393.

Torrence, C., and G. P. Compo, 1998: A practical guide to wavelet analysis. Bull. Amer. Meteor. Soc., 79, 61-78, https://doi.org/ 10.1175/1520-0477(1998)079<0061:APGTWA>2.0.CO;2.

van Haren, H., 2004: Incoherent internal tidal currents in the deep ocean. Ocean Dyn., 54, 66-76, https://doi.org/10.1007/ s10236-003-0083-2.

_ L. Maas, J. Zimmerman, H. Ridderinkhof, and H. Malschaert, 1999: Strong inertial currents and marginal internal wave stability in the central North Sea. Geophys. Res. Lett., 26, 2993-2996, https://doi.org/10.1029/1999GL002352.

— M. Me Jong, and P. Kooijman, 2015: Yearlong moored bioluminescence and current data at KM3NeT neutrino telescope sites in the deep Ionian Sea. Astropart. Phys., 67, 1-7, https:// doi.org/10.1016/j.astropartphys.2015.01.005.

Vlasenko, V., N. Stashchuk, C. Guo, and X. Chen, 2010: Multimodal structure of baroclinic tides in the South China Sea. Nonlinear Processes Geophys., 17, 529-543, https://doi.org/ 10.5194/npg-17-529-2010.

Wahba, G., 1985: A comparison of GCV and GML for choosing the smoothing parameter in the generalized spline smoothing problem. Ann. Stat., 13, 1378-1402, https://doi.org/10.1214/aos/1176349743.

Wu, L., C. Miao, and W. Zhao, 2013: Patterns of $\mathrm{K}_{1}$ and $\mathrm{M}_{2}$ internal tides and their seasonal variations in the northern South China Sea. J. Oceanogr., 69, 481-494, https://doi.org/ 10.1007/s10872-013-0183-7.

Xu, Z., B. Yin, and Y. Hou, 2011: Multimodal structure of the internal tides on the continental shelf of the northwestern South
China Sea. Estuarine Coastal Shelf Sci., 95, 178-185, https:// doi.org/10.1016/j.ecss.2011.08.026.

, and Y. Xu, 2013: Variability of internal tides and near-inertial waves on the continental slope of the northwestern South China Sea. J. Geophys. Res. Oceans, 118, 197-211, https://doi.org/10.1029/2012JC008212.

—, K. Liu, B. Yin, Z. Zhao, Y. Wang, and Q. Li, 2016: Longrange propagation and associated variability of internal tides in the South China Sea.J. Geophys. Res. Oceans, 121, 8268-8286, https://doi.org/10.1002/2016JC012105.

Zaron, E. D., and D. A. Jay, 2014: An analysis of secular change in tides at open-ocean sites in the Pacific. J. Phys. Oceanogr., 44, 1704-1726, https://doi.org/10.1175/JPO-D-13-0266.1.

Zhang, J., and X. Lu, 2010: Inversion of three-dimensional tidal currents in marginal seas by assimilating satellite altimetry. Comput. Methods Appl. Math. Eng., 199, 3125-3136, https:// doi.org/10.1016/j.cma.2010.06.014.

,$- \ldots$, P. Wang, and Y. P. Wang, 2011: Study on linear and nonlinear bottom friction parameterizations for regional tidal models using data assimilation. Cont. Shelf Res., 31, 555-573, https://doi.org/10.1016/j.csr.2010.12.011.

Zhang, Z., O. Fringer, and S. Ramp, 2011: Three-dimensional, nonhydrostatic numerical simulation of nonlinear internal wave generation and propagation in the South China Sea. J. Geophys. Res., 116, C05022, https://doi.org/10.1029/2010JC006424.

—, W. Zhao, J. Tian, and X. Liang, 2013: A mesoscale eddy pair southwest of Taiwan and its influence on deep circulation. J. Geophys. Res. Oceans, 118, 6479-6494, https://doi.org/10.1002/ 2013JC008994.

Zhao, Z., M. H. Alford, J. A. MacKinnon, and R. Pinkel, 2010: Long-range propagation of the semidiurnal internal tide from the Hawaiian Ridge. J. Phys. Oceanogr., 40, 713-736, https:// doi.org/10.1175/2009JPO4207.1.

Zu, T., J. Gan, and S. Y. Erofeeva, 2008: Numerical study of the tide and tidal dynamics in the South China Sea. Deep-Sea Res. I, 55, 137-154, https://doi.org/10.1016/j.dsr.2007.10.007. 